\title{
Asymptotic theory for a Leidenfrost drop on a liquid pool
}

\author{
Michiel A. J. van Limbeek ${ }^{1, \dagger}$, Benjamin Sobac ${ }^{2}$, Alexey Rednikov ${ }^{2}$, \\ Pierre Colinet ${ }^{2}$ and Jacco H. Snoeijer ${ }^{1}$ \\ ${ }^{1}$ University of Twente, Physics of Fluids group, P.O. Box 217, 7500AE Enschede, The Netherlands \\ ${ }^{2}$ Université Libre de Bruxelles, TIPs - Fluid Physics, C.P. 165/67, Av. F. D. Roosevelt 50, 1050 \\ Brussels, Belgium
}

(Received 31 May 2018; revised 18 October 2018; accepted 18 December 2018; first published online 29 January 2019)

Droplets can be levitated by their own vapour when placed onto a superheated plate (the Leidenfrost effect). It is less known that the Leidenfrost effect can likewise be observed over a liquid pool (superheated with respect to the drop), which is the study case here. Emphasis is placed on an asymptotic analysis in the limit of small evaporation numbers, which indeed proves to be a realistic one for millimetric-sized drops (i.e. where the radius of the drop is of the order of the capillary length). The global shapes are found to resemble 'superhydrophobic drops' that follow from the equilibrium between capillarity and gravity. However, the morphology of the thin vapour layer between the drop and the pool is very different from that of classical Leidenfrost drops over a flat rigid substrate, and exhibits different scaling laws. We determine analytical expressions for the vapour thickness as a function of temperature and material properties, which are confirmed by numerical solutions. Surprisingly, we show that deformability of the pool suppresses the chimney instability of Leidenfrost drops.

Key words: condensation/evaporation, drops, lubrication theory

\section{Introduction}

A drop can be prevented from merging with a liquid bath when the bath is heated above the saturation temperature. Recently, such Leidenfrost drops have regained attention (Maquet et al. 2016) after the first reports by Hickman (1964b), who more than half a century ago referred to these drops as 'boules'. Figure 1 gives an example of such a large water drop that is prevented from contacting a pool of water. The evaporation gives rise to a thin vapour layer between the drop and the pool, and the corresponding vapour flow induces a pressure that keeps the drop separated from the pool.

Naturally, one tries to compare these 'boules' to drops levitated above a heated plate. The latter have been studied in great detail (Wachters, Bonne \& van Nouhuis

$\dagger$ Email address for correspondence: m.a.j.vanlimbeek@utwente.nl 




FIgURE 1. A Leidenfrost drop of water floating on a water bath that is heated to a few degrees above the saturation temperature. The drop, also referred to as a 'boule', has a radius of $7 \mathrm{~cm}$, which is 25 times the capillary length. (Reprinted with permission from Hickman (1964b) 'Floating drops and liquid boules'. Copyright 2016 American Chemical Society.)

1966; Bernardin \& Mudawar 1999; Biance, Clanet \& Quéré 2003; Quéré 2013) since the first report by Leidenfrost (1756) centuries ago. Since then various studies have focussed on features as shape oscillations (Holter \& Glasscock 1952; Ryuji \& Ken 1985; Strier et al. 2000; Snezhko, Ben Jacob \& Aranson 2008; Brunet \& Snoeijer 2011; Bouwhuis et al. 2013; Ma, Liétor-Santos \& Burton 2017), drop mobility on ratchets or gradients (Linke et al. 2006; Lagubeau et al. 2011; Würger 2011; Sobac et al. 2017), dynamics during drop impacts (Chandra \& Avedisian 1991; Tran et al. 2012; Shirota et al. 2016) and the Leidenfrost temperature (Baumeister \& Simon 1973; van Limbeek et al. 2016).

Of particular interest is the shape of such Leidenfrost drops. When viewed from the side, a Leidenfrost drop above a plate resembles a sessile drop that makes a contact angle of $180^{\circ}$ with the substrate (Biance et al. 2003; Snoeijer, Brunet \& Eggers 2009; Quéré 2013; Sobac et al. 2014). The vapour layer prevents a direct contact so that the droplet is maintained in a perfectly non-wetting, 'superhydrophobic' state. Intriguingly, it is only fairly recently that the morphology of the thin vapour layer below the drop has been revealed. Experimentally, the shape was characterised by interferometry by Burton et al. (2012), showing that the thickness of the vapour layer is not uniform. This is sketched in figure 2(a), where one observes a large vapour pocket near the centre of the drop and a thin 'neck' near its edge. For large drop radii, the base of the drop can even penetrate up to the top, enabling vapour to escape by a 'chimney instability' (Biance et al. 2003; Snoeijer et al. 2009). Even prior to experiments, the details of the layer below levitated drops were predicted from a hydrodynamic analysis (Duchemin, Lister \& Lange 2005; Lister et al. 2008; Snoeijer et al. 2009), with a more complete description of the evaporation developed by Sobac 
(a)

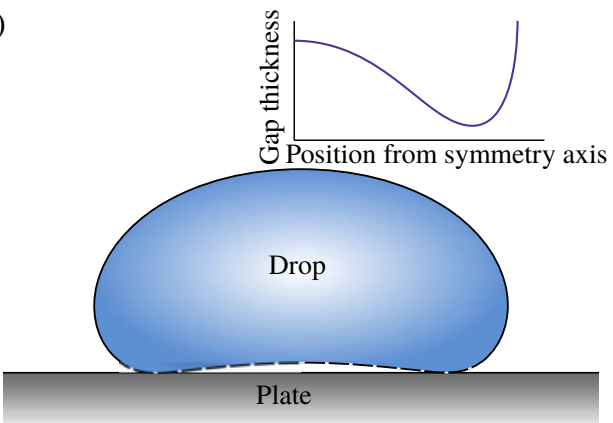

(b)



FIGURE 2. (Colour online) Sketches of a Leidenfrost drop levitated above a hot plate (a) and above a hot pool $(b)$ based on numerical simulations. The resulting shapes are essentially 'superhydrophobic drops' (solid lines), with an underlying thin vapour layer (dashed lines). The insets provide a detailed zoom of the geometry of the vapour layer, revealing a striking difference between the two cases.

et al. (2014). In the limit of small evaporation numbers (a capillary number based on an evaporation velocity scale), one indeed finds the neck to be asymptotically thinner than the vapour film at the centre, and scaling laws characterising such vapour pockets were established. One of the salient features is that the vapour pressure that carries the weight of the drop is nearly uniform below the drop: owing to the viscous resistance to vapour flow, the pressure falls abruptly across the thin neck to reach the atmospheric pressure.

Leidenfrost drops on a pool exhibit very different morphologies (Maquet et al. 2016). A typical numerical result is shown in figure $2(b)$. Globally the drop can still be considered in a 'superhydrophobic' state, but now on a deformable pool rather than on a flat substrate. The shape of the vapour layer, however, is very different from Leidenfrost drops on such a substrate: the vapour layer is nearly uniform and exhibits oscillations before passing a thin neck. A strong connection can be made to the film drainage problem for drop coalescence (Jones \& Wilson 1978; Yiantsios \& Davis 1990). In that context, a drop is floating on a pool as well while the gravity forces the two bodies to merge eventually. Although the film drains over time, and is not replenished by evaporation, the deformability of the pool results in a thin gas layer (Jones \& Wilson 1978) with a morphology reminiscent of those found for the Leidenfrost drop. Also, the numerical analysis of Maquet et al. (2016) showed no indication of a chimney instability for Leidenfrost drops on a pool, even for drops considerably larger than the capillary length. The difference in gap spacing is importance as it determines the vapour generation.

Note that the case by Maquet et al. (2016) is actually slightly different from the boule case by Hickman (1964b) in the following regard. In the former (Leidenfrost) case, the evaporative heat flux is limited by heat conduction across the vapour gap from the superheated pool surface (non-volatile) to the drop surface being maintained at the saturation temperature, evaporation proceeding from the drop surface. In the latter (boule) case, the two liquids being the same, both surfaces of the vapour gap find themselves at the same, saturation temperature, while the evaporative heat flux is rather limited by heat transport from the superheated bulk of the pool, with evaporation eventually taking place from the surface of the pool. 
In the present paper, a baseline consideration will explicitly be adapted to the former case (Maquet et al. 2016), whereas the boule case will be only mimicked by choosing equal densities and surface tensions of the two liquids. While it is just a numerical solution of the problem that was provided by Maquet et al. (2016) in the theoretical part of their work, here we aim at a detailed exploration of the physics and structure of the phenomenon by means of asymptotic methods.

Our investigation of Leidenfrost drops on a pool will be based on a matched asymptotics analysis in the limit of small evaporation numbers where the vapour generation vanishes. We compute the detailed structure of the vapour layer as in figure 2, and establish the scaling laws for the thickness as a function of the material properties and the superheat. In $\S 2$ we formulate the problem and sketch the asymptotic structure, which is worked out in detail in $\S 3$. The boules of Hickman are discussed at the end of that section. Analytical results are obtained in the limit of large drops, explaining why, indeed, there is no chimney instability above a pool. The results are generalised in $\S 4$ for the case of smaller drops and differing liquids, showing that the scaling laws are robust. The paper closes with a discussion in $\S 5$.

\section{Formulation}

In this section we first present a set of equations that describe a steady Leidenfrost drop levitated above a liquid pool. This part follows the ideas presented in Maquet et al. (2016), although the problem is formulated in terms more amenable to our present analysis. We then sketch how the equations are solved by means of matched asymptotic expansions.

\subsection{Model}

We first need to establish a convenient representation of the drop-on-pool geometry shown in figure $2(b)$. The problem consists of two axisymmetric liquid domains, the drop and the pool, which we describe by the position of their respective liquid-vapour interfaces $z=h$ (for the drop) and $z=e$ (for the pool), where the $z$ axis points vertically upwards with $z=0$ corresponding to the unperturbed pool surface far away from the drop. These are defined in more detail in figure 3. While $h$ and $e$ in principle provide a full description of the geometry, we also introduce the thickness of the vapour layer $t$. This is convenient for describing the flow inside the thin vapour layer below the drop, where the two interfaces are essentially parallel.

Following previous theoretical developments on levitated drops (Jones \& Wilson 1978; Lister et al. 2008; Snoeijer et al. 2009; Sobac et al. 2014; Maquet et al. 2016), we consider the upper surfaces of the liquid drop and pool to be at hydrostatic equilibrium while the flow of the produced vapour is treated in the lubrication approximation. We first compute the vapour pressure $P_{v}$ by approaching it from the side of the pool,

$$
P_{v}=-\rho_{p} g e+\gamma_{p} \kappa_{e}
$$

Here we introduced the pool density $\rho_{p}$ and surface tension $\gamma_{p}$, while the $\kappa_{e}$ is the curvature of the pool interface. The first term represents the hydrostatic pressure inside the pool, while the curvature term is the Laplace pressure jump due to surface tension. Note that the hydrostatic pressure inside the pool was taken to be $-\rho_{p} g e$, i.e. the atmospheric pressure was set to zero. Similarly, we obtain an expression for the $P_{v}$ from the side of the drop

$$
P_{v}=k-\rho_{d} g h-\gamma_{d} \kappa_{h},
$$




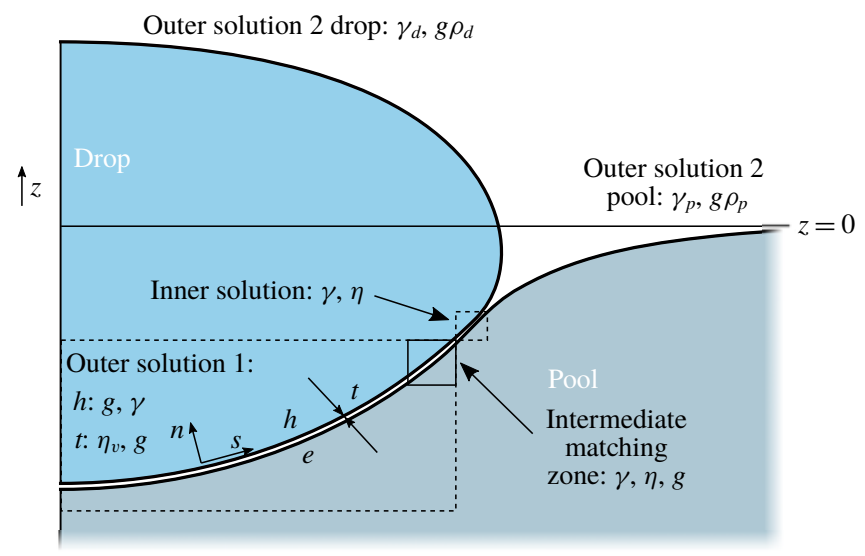

FIGURE 3. (Colour online) Sketch of a drop on a pool. Different zones are identified which are used for the asymptotic analysis for $\mathcal{E} \ll 1, \mathcal{R} \gg 1$ for identical fluid properties $(\Gamma=1, \mathcal{P}=1)$. The dominant force balances in the inner/outer regimes are indicated as capillary $(\gamma)$, viscous $\left(\eta_{v}\right)$ and gravitational $(g)$. Also defined are the vapour-layer thickness $t$, which is the separation between the drop interface $h$ and the pool interface $e$, and $s$ and $n$, which denote the orientation of the curvilinear coordinate system. Note that in the case of non-equal properties the thickness is also determined by the mechanical properties of both the drop and pool denoted by the subscripts ' $d$ ' for drop and ' $p$ ' for pool.

where $\rho_{d}, \gamma_{d}, \kappa_{h}$ represent the droplet density, surface tension and curvature and $k$ is an integration constant. Equations (2.1), (2.2) give two separate expressions for $P_{v}$, which in the lubrication approximation for thin layers must be identical. Therefore (2.1), (2.2) can also be seen as a relation between $h$ and $e$.

We now turn to the flow inside the vapour layer. It will be shown that for sufficiently small evaporation rates the gap thickness $t$ is asymptotically small, justifying the use of the lubrication theory. We therefore consider the reduced Stokes equation for the parallel velocity $u$,

$$
\partial_{s} P_{v}=\eta_{v} \partial_{n n} u
$$

where $s$ is the curvilinear coordinate along the layer, while $n$ is the coordinate perpendicular to the vapour film (see figure 3). Owing to the small gas viscosity $\eta_{v}$, we here assume that no flow is induced inside the drop and the pool. As a consequence, we can solve (2.3) with no-slip boundary conditions at $n=0$ and $n=t$, yielding a parabolic profile

$$
u=6 \bar{u}\left(\frac{n}{t}-\frac{n^{2}}{t^{2}}\right),
$$

where we introduced the thickness-averaged velocity

$$
\bar{u}=-\frac{t^{2} \partial_{s} P_{v}}{12 \eta_{v}} .
$$

Note that for an infinite drop slip could occur despite the significant viscosity contrast, since the ratio between the drop and film thickness is also large. In that case, the 
factor 1/12 changes, depending on the slip length. Only a fully resolved numerical simulation of the Navier-Stokes equations in drop, film and pool will give insight into this effect, which is beyond the scope of this study. Here we stick with the no-slip assumption, frequently used in the literature (Wachters et al. 1966; Hinch \& Lemaitre 1994; Quéré 2013; Maquet et al. 2016; Janssens, Koizumi \& Fried 2017).

The lubrication problem is closed using the axisymmetric continuity equation,

$$
r \dot{t}+\partial_{s}(r t \bar{u})=r j
$$

where $r$ is the distance from the symmetry axis and $\dot{t}$ is a time derivative; in the remainder we will look for (quasi-)steady states (the evaporation time of the drop being much greater than the relaxation time of an initially deposited drop to its quasisteady shape and in particular the viscous relaxation time in the vapour layer) so that time derivatives can be omitted. The source term $j$ on the right-hand side of (2.6) is due to the flux of vapour generated by evaporation, which modelled by Fourier's law can be expressed as (Maquet et al. 2016)

$$
j=\frac{\epsilon}{t} \quad \text { with } \epsilon=\frac{k_{v} \Delta T}{L \rho_{v}} .
$$

In this expression $k_{v}$ and $\rho_{v}$ respectively are the vapour thermal conductivity and density, $L$ the latent heat of evaporation and $\Delta T$ the temperature difference between the pool and the drop (the superheat). The result deviates here from the description of the drainage problem: while the source term is absent in the drainage problem, similar effects occur due to the thinning of the gap driving the gas flow. Note that in the case of the boules of Hickman, the pool is superheated and evaporating, which can be modelled using Newton's law of cooling: $j=\hbar \Delta T /\left(L \rho_{v}\right)$, where $h$ (superheated) pool temperature far away from the drop (Bejan 1993). In this case, $j$ is approximately constant along the film. For now, we focus on the Leidenfrost case of (2.7). The consequences of this different mechanism of vapour generation will be discussed in $(\S 3.5)$.

Thus, the vapour film is described by

$$
-\frac{1}{12 \eta_{v} r} \partial_{s}\left(r t^{3} \partial_{s} P_{v}\right)=\frac{\epsilon}{t}
$$

in conjunction with (2.1) and (2.2), which are three coupled equations for the vapour pressure $P_{v}$ and respectively for the droplet and pool surface profiles $h$ and $e$. These equations need to be complemented by geometric expressions (see appendix $\mathrm{A}$ for more details) for the interface curvatures $\kappa_{e, h}$, for $r$ as a function of $s, v i z .\left(\partial_{s} e\right)^{2}+$ $\left(\partial_{s} r\right)^{2}=1$, and for the thickness of the vapour layer, viz.

$$
t(s) \partial_{s} r=h(s)-e(s) .
$$

At the exit from the vapour film, where it joins the ambient atmosphere and where its thickness $t$ asymptotically diverges, the drop and pool surfaces are expected to attain equilibrium static shapes, described by

$$
P_{v}=0
$$

with (2.2) and (2.1), respectively. It may be useful to regard (2.10) as a degenerate form of (2.8) as $t \rightarrow \infty$. It is matching with such static shapes that is imposed in one way or another (to be specified at each concrete occurrence) as the boundary conditions there. 
As for other boundary conditions, no singularity at the symmetry axis (i.e. at $r=0$ ) is imposed. Finally, the pool surface attains its unperturbed level $e=0$ far away from the drop (as $r \rightarrow \infty$ ).

The constant parameter $k$ entering the problem by means of (2.2) is eventually the one quantifying the size of the droplet, and in this sense could be taken as one of the system parameters in the analysis (along with the material properties of the liquids and the superheat). However, we shall rather prefer to quantify the size more directly by means of the radius of the droplet's vertical projection, $R$. Hence, with $R$ as a system parameter, $k$ now becomes yet another unknown to be determined. Most cases however yield no closed form for $R(k)$, hence $k$ is used in the numerical calculations to converge the problem towards a certain desired drop size $R$.

For the time being, we shall keep the formulation in dimensional form. Appropriate non-dimensionalisations will rather be introduced later on in a context-specific way. However, one can already establish that the results are ultimately governed by four dimensionless parameters, which can be chosen as

$$
\Gamma=\frac{\gamma_{p}}{\gamma_{d}}, \quad \mathcal{P}=\frac{\rho_{p}}{\rho_{d}}, \quad \mathcal{R}=\frac{R}{\lambda_{c}}, \quad \tilde{\mathcal{E}}=\frac{\eta_{v} \epsilon}{\rho_{d} g \lambda_{c}^{3}} .
$$

The first two are the ratios of surface tension and density of the pool and the liquid. The third parameter $\mathcal{R}$ is the dimensionless radius of the drop, scaled by the capillary length $\lambda_{c}=\left(\gamma_{d} / \rho_{d} g\right)^{1 / 2}$. Finally, the evaporation-induced viscous vapour flow is quantified by the evaporation number $\tilde{\mathcal{E}}$, proportional to the value of the superheat. Note that a key dimensionless number $\tilde{\mathcal{E}}$ naturally appears upon substituting (2.2) into (2.8) and normalising all the length variables with $\lambda_{c}$ (Sobac et al. 2014; Maquet et al. 2016).

\subsection{Asymptotic approach}

The values of the evaporation number $\tilde{\mathcal{E}}$ encountered in practice are typically rather small (Snoeijer et al. 2009; Celestini, Frish \& Pomeau 2012; Sobac et al. 2014; Maquet et al. 2016). This is what eventually justifies the very structure of the problem assumed in $\S 2.1$ : a thin vapour layer between the substrate (here the pool), where the lubrication approximation is applicable, and equilibrium shapes of liquid surfaces (drop and pool) beyond the thin vapour layer. Direct computations of the Leidenfrost problem carried out under this premise confirm its self-consistency for both flat solid substrates (Snoeijer et al. 2009; Sobac et al. 2014) and deformed liquid ones (Maquet et al. 2016). Essential deviations from this scheme are only encountered, on the one hand, for sufficiently small drops, well below the capillary length (Celestini et al. 2012; Sobac et al. 2014). In the present paper, we shall deal only with drops larger than this (see below), and hence encounter no such limitation. On the other hand, the scheme breaks down on the verge of the chimney instability (Snoeijer et al. 2009; Sobac et al. 2014), which, as already anticipated, will not be encountered in the present case of a liquid substrate the same mechanical properties as the drop.

Thus, in fact, the formulation provided in $\S 2.1$ already tacitly implies $\tilde{\mathcal{E}} \ll 1$. Direct numerical computation for such a 'full' formulation can be realised e.g. using an approach largely similar to Sobac et al. (2014) and Maquet et al. (2016). Here it is just rendered geometrically more elaborate in order to handle large deformations of the pool surface, see appendix A for more details. 
However, $\tilde{\mathcal{E}} \ll 1$ also opens the way to a systematic asymptotic analysis, consistently and thoroughly exploiting this limit. This is actually what the present paper is about. As is typically the case, such an asymptotic analysis will permit further insight into the physics of the problem. Here we shall in particular be interested in further details as far as the structure of the vapour layer is concerned. Importantly, this will also permit us to establish the scaling with $\tilde{\mathcal{E}}$ of various quantities of interest. In the case of a flat solid substrate, such a program has been realised e.g. by Snoeijer et al. (2009) and Sobac et al. (2014). As already stated, we anticipate essential differences in the case of the liquid substrate we are concerned with in the present paper. The hereby obtained asymptotic results will be validated against the direct numerical simulation mentioned earlier in the framework of the full formulation of $\S 2.1$, while highlighting similarities and differences with the description of the drainage problem.

With the expected asymptotically small vapour-layer thickness $t$ in the limit of small evaporation numbers $\tilde{\mathcal{E}} \ll 1$, one actually realises that the shape of our Leidenfrost drop must be asymptotically close to that of an equilibrium 'superhydrophobic' drop. The shape of the latter is governed by the static balance between surface tension and gravity. Namely, in this superhydrophobic configuration, the liquid-liquid interface is determined by (2.1) and (2.2) with $e \equiv h$ and formally possesses an interfacial tension $\gamma_{d p} \equiv \gamma_{p}+\gamma_{d}$; the liquid-gas interface is described by (2.2) with (2.10) for the drop itself, and by (2.1) with (2.10) for the pool; at the contact (triple) line, the slopes of all the three interfaces coincide, and a contact angle of $180^{\circ}$ results from the side of each of the liquids (see appendix B for the computation method). It is important to note that the picture in terms of a superhydrophobic drop refers to a large-scale description: in the actual non-equilibrium configuration, the 'contact line' in fact represents the position of a thin neck region through which the vapour escapes to the surrounding atmosphere.

Once the superhydrophobic shape is known, the leading-order pressure distribution governing the vapour flow in (2.8) can immediately be drawn from (2.1), or equivalently from (2.2). This distribution will clearly not be constant in the pool case, with an appreciably non-flat surface, i.e. we end up with $\partial_{s} P_{v} \neq 0$ independently (to leading order) of the actual vapour-layer profile $t(s)$. This is in stark contrast to a drop on a flat rigid substrate, for which $\partial_{s} P_{v} \neq 0$ does depend upon $t(s)$ (i.e. upon a higher-order approximation in terms of $\tilde{\mathcal{E}} \ll 1$ ). We shall see that this is the key factor giving rise to different vapour-layer structures and scalings for the two types of substrate, typical for drops on deformable surfaces (Jones \& Wilson 1978; Maquet et al. 2016).

However, a common feature between the two types of substrate is that $P_{v}$ will not be continuous across the 'triple line', i.e. across the thin neck region through which the vapour escapes from below the drop. This is due to a mismatch in curvature on both sides of the neck, where $P_{v}$ exhibits a jump from $P_{v}>0$ under the drop to the ambient value $P_{v}=0$. In experiments however $P_{v}$ will be continuous, which will be discussed in more detail in $\$ 3.2 .2$. The appearance of this thin neck allows us to introduce and exploit the following hierarchy of length scales

$$
t_{n} \ll t_{0} \ll \lambda_{c} \sim R,
$$

where $t_{0}$ and $t_{n}$ are, respectively, the typical vapour layer thicknesses outside and inside the neck region. Such a presence of separate distinguished regions with different spatial scales means that we resort to matched asymptotic expansions as the most appropriate asymptotic method for the problem at hand. Overall, it turns out that the 
problem can be split into the following regions, indicated schematically in figure 3 . At large scales, there are two outer regions: region 1 below the drop, and region 2 above the drop and pool. In the limit of vanishing $t$, these outer solutions will precisely correspond to superhydrophobic drops on a liquid pool. As already mentioned, these are equilibrium solutions that can be computed from the static balance between surface tension and gravity. These two outer regions are connected by a smaller inner region, which is nothing other than the earlier mentioned neck region. It will turn out that no direct matching between the outer and inner regions is possible in the vapour layer, and so yet another, intermediate region is implied, which is also marked in figure 3.

As already pointed out, the expected completely new type of solution and the $\tilde{\mathcal{E}}$-scalings for the vapour layer basically owe themselves to the substrate surface deformability. Note however that, for smaller drops, $R<\lambda_{c}(\mathcal{R}<1)$, the pool surface becomes increasingly flat (Maquet et al. 2016). Therefore, for sufficiently small drops, the appropriate asymptotic theory is likely to involve other smallness parameters apart from $\tilde{\mathcal{E}}$, viz. a geometric one characterising the small substrate non-flatness. Another kind of limitation for smaller drops and related to the mentioned intermediate region will be pointed out in $\S 4.1$. However, in essence, we shall consider drops that are not sufficiently large to be beyond the scope of the present paper.

Furthermore, a large part of our analysis ( $\$ 3)$ will be dedicated to very large drops, $R \gg \lambda_{c}(\mathcal{R} \gg 1)$. The expected hierarchy of length scales (2.12) then rewrites as

$$
t_{n} \ll t_{0} \ll \lambda_{c} \ll R .
$$

Besides, inspired by the boules of Hickman (1964a), we launch the analysis by considering liquids with equal properties, $\Gamma=1$ and $P=1$. Mathematically, this gives rise to the simplest possible configuration (the associated superhydrophobic drop assuming a hemispherical shape), where analytical solutions are possible and which is a good starting point for developing the essence of our asymptotic approach. Subsequently, in $\S 4$, we extend our analysis to smaller drops, $R \sim \lambda_{c}(\mathcal{R} \sim 1)$, and to non-equal liquid properties, $\Gamma \neq 1$ and/or $\mathcal{P} \neq 1$.

\section{A large drop on a pool with the same mechanical properties}

Here we perform a detailed analysis of a situation resembling the 'boules' of Hickman (1964b), shown in figure 1. In this case the pool and the drop have equal density and surface tension, i.e. $\Gamma=\mathcal{P}=1$. The boules formed are much larger than the capillary length, $\mathcal{R} \gg 1$, and below we will consider the asymptotics for small evaporation numbers, $\mathcal{E} \ll 1$.

\subsection{Outer region 1: below the drop}

\subsubsection{The droplet shape}

The outer shape of the drop and pool can be obtained by combining (2.1) and (2.2):

$$
0=k-\rho g(h-e)-\gamma\left(\kappa_{h}+\kappa_{e}\right),
$$

where we assumed identical material properties $\gamma=\gamma_{d}=\gamma_{p}$ and $\rho=\rho_{d}=\rho_{p}$. We anticipate the gap thickness, $t \sim h-e$, to be much smaller than the radius, in which case $\kappa_{h} \simeq \kappa_{e}$. The hydrostatic term can also be neglected when $t \sim(h-e) \ll 4 \gamma / \rho g R \sim$ $\lambda_{c}^{2} / R$, a condition that is much more severe and will be monitored for our solution $a$ posteriori. Equation (3.1) then further simplifies,

$$
0=k-2 \gamma \kappa_{h},
$$


(a)

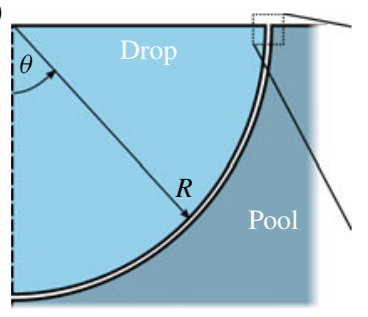

(b)



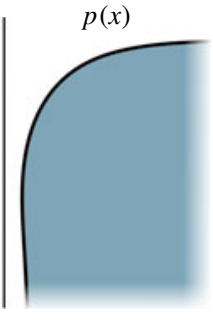

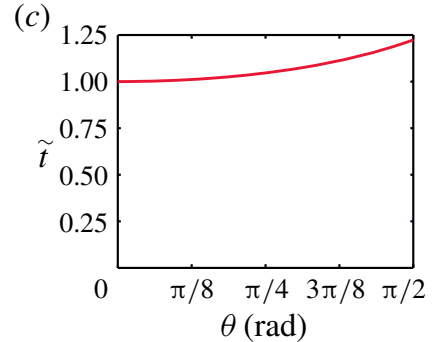

FIGURE 4. (Colour online) A sketch of outer region 1 is shown in $(a)$. Since the shape below the drop reduces to a hemisphere, we adopt a spherical coordinate system where the gap thickness $t$ depends on the angle $\theta$. (b) The local Cartesian coordinate system around $\theta=\pi / 2$, which is used for the analysis of the thin neck region at the exit of the vapour layer. The drop and pool interfaces are expressed as $d(x)$ and $p(x)$ respectively, while the vapour thickness reads $t=d-p$. The vapour thickness profile (3.9), non-dimensionalised using the scale of (3.5), is represented in $(c)$.

imposing a shape of constant curvature. Below we will find that the matching condition requires the outer solution to be a perfect hemisphere. Hence, we can identify $k=4 \gamma / R$, where $R$ is the maximum radius of the drop.

\subsubsection{The vapour thickness}

The spherical geometry of the outer solution, and thus of the vapour layer, suggests that the analysis of the lubrication flow will be most easily expressed using spherical coordinates (see figure 4). In this coordinate system, the lubrication equation (2.8) reads

$$
-\frac{1}{12 \eta_{v} R^{2} \sin \theta} \partial_{\theta}\left[\begin{array}{lll}
\sin \theta & t^{3} & \partial_{\theta} P_{v}
\end{array}\right]=\frac{\epsilon}{t} .
$$

This equation needs to be complemented by an equation for the pressure gradient $P_{v}^{\prime}$, for example (2.2), which in spherical coordinates simplifies to $\partial_{\theta} P_{v}=-\rho g R \sin \theta$. The lubrication equation can then be expressed as

$$
\frac{1}{2 t^{3} \sin \theta} \partial_{\theta}\left[t^{3} \sin ^{2} \theta\right]=\frac{6 \eta_{v} \in R}{\rho g t^{4}} .
$$

In this expression we have collected all dimensional parameters on the right-hand side, to form a dimensionless ratio.

The above expression invites us to introduce a dimensionless thickness $\tilde{t}=t / t_{*}$, where the characteristic scale for the thickness reads

$$
t_{*}=\left(\frac{6 \eta_{v} R \epsilon}{\rho g}\right)^{1 / 4}=\lambda_{c} \mathcal{E}^{1 / 4},
$$

where a (modified) evaporation number

$$
\mathcal{E}=\frac{6 \eta_{v} R \epsilon}{\rho_{d} g \lambda_{c}^{4}}=\frac{6 R}{\lambda_{c}} \tilde{\mathcal{E}}
$$


has been introduced, as it naturally occurs in this form in the present $\mathcal{R} \gg 1$ context. Inserting this rescaling into (3.4) and working out the derivatives gives

$$
\tilde{t}^{3} \partial_{\theta} \tilde{t}=\frac{2\left[1-\tilde{t}^{4} \cos \theta\right]}{3 \sin \theta},
$$

which is a first-order ordinary differential equation (ODE) for the profile of the vapour layer $\tilde{t}(\theta)$. The solution to this equation can be cast in closed form

$$
\begin{aligned}
{[\tilde{t}(\theta)]^{4}=} & \frac{8 \int_{0}^{\theta} \mathrm{d} x(\sin x)^{5 / 3}}{3(\sin \theta)^{8 / 3}}=\frac{8}{15} \frac{1}{(\sin \theta)^{8 / 3}} \\
& \times\left(\frac{\pi^{1 / 2} \Gamma\left(\frac{1}{3}\right)}{\Gamma\left(\frac{5}{6}\right)}-\cos \theta\left(2{ }_{2} \mathrm{~F}_{1}\left[\frac{1}{2}, \frac{2}{3}, \frac{3}{2},(\cos \theta)^{2}\right]+3(\sin \theta)^{2 / 3}\right)\right) .
\end{aligned}
$$

Here, we imposed a non-singular behaviour at the symmetry axis, only possible with $\tilde{t}_{0} \equiv \tilde{t}(0)=1$, which was anticipated in the definition in (3.5). Here $\Gamma(x)$ is the gamma function, ${ }_{2} F_{1}$ is a generalised hypergeometric function (Weisstein 2017) and this expression was obtained using Mathematica. The functional form is different here compared to the film drainage problem, as the present source term has a $1 / t$ dependence, while in the latter case it is a function of $\theta$ (Jones \& Wilson 1978). When plotting the solution (3.8), one observes that the vapour thickness is nearly constant, see figure $4(c)$. It very mildly increases from $\tilde{t}=1$ at $\theta=0$, to a slightly larger value at $\theta=\pi / 2$ (the exit of the gap):

$$
\tilde{t}_{\text {exit }}=2^{3 / 4} 15^{-1 / 4} \pi^{1 / 8}\left(\frac{\Gamma\left(\frac{1}{3}\right)}{\Gamma\left(\frac{5}{6}\right)}\right)^{1 / 4} \approx 1.22386 \cdots .
$$

Comparing the vapour film with that of a drop on a heated plate, we find that the film only mildly changes towards the free atmosphere. For a drop on a plate, a much stronger scaling was found for the dependency of the vapour thickness, depending on both the radius and evaporation number (Sobac et al. 2014). As a result, the global evaporation rate is much higher for the present case as the drop is close to the heat source throughout the vapour gap.

\subsubsection{Summary and comparison to numerical solution}

An important set of analytical results has thus been obtained. First, we found that the immersed part of the drop takes a hemispherical shape. Second, the vapour-layer thickness at the axis below the drop is given by $\tilde{t}_{0}=1$. Third, the profile of the vapour layer in the outer zone is characterised by a single, universal profile, given by the expression (3.8). For the matching to the neck region (see figure 4), an important result is the thickness at the 'gap exit' at $\theta=\pi / 2$, for which the analytical expression (3.9) is found. In dimensional form, we thus obtain the relevant thicknesses

$$
t_{0}=\lambda_{c} \mathcal{E}^{1 / 4}, \quad t_{\text {exit }}=1.22386 \cdots \lambda_{c} \mathcal{E}^{1 / 4} .
$$

These results were obtained under the assumption of the hierarchy of scales (2.13), which is self-consistent as long as

$$
\mathcal{E}^{1 / 4} \ll 1 \ll \mathcal{R} \ll \mathcal{E}^{-1 / 4}
$$




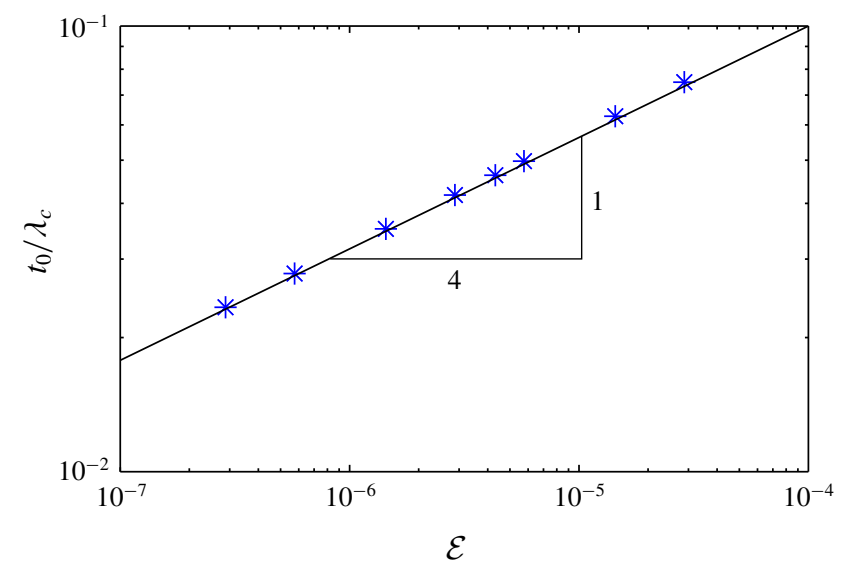

FIgURE 5. (Colour online) Results for the central thickness $t_{0} \equiv t(0)$ of the vapour layer versus the evaporation number for a drop of $R=10 \lambda_{c}$ on a pool having the same properties as the drop. The data points (numerical solution to the full problem) approach the curve $t_{0}=\lambda_{c} \mathcal{E}^{1 / 4}$ obtained by asymptotic methods in the limit of small $\mathcal{E}$.

Note the latter, strong inequality arising from neglecting the hydrostatic pressure difference across the vapour gap $\left(t \ll \lambda_{c}^{2} / R\right)$. To validate these findings, we compared the results to numerical solutions of the full problem formulated in $\S 2.1$ (see also appendix A). Indeed, it is observed that for large drop volumes the immersed part of the droplet approaches a perfect hemisphere. In figure 5 we provide a further quantitative test, by plotting the central thickness $t_{0}$ for a droplet of radius $R=10 \lambda_{c}$. Upon reducing the evaporation number $\mathcal{E}$, the numerical result perfectly approaches the prediction (3.10). Moreover, note that the last inequality (3.11) is well satisfied in the given range of $\mathcal{E}$. Evaluating $t_{0}$ for the experimental conditions of figure 1 , we obtain $t_{0} \approx 70 \mu \mathrm{m}$.

\subsection{Outer region 2}

\subsubsection{Puddle solutions}

We now turn to the second outer region, describing the top of the droplet and of the pool. In this range the vapour is no longer confined to a thin gap, so that viscous effects are completely negligible here. As a consequence, the pool and drop are described by (2.1) and (2.2) with $P_{v}=0$. We further note that the constant $k \rightarrow 0$ for large drops. This implies that, even though the azimuthal curvature has the opposite sign for the drop and for the pool, its contribution is negligible. Therefore, the profiles of the drop and pool become mirror images of each other.

The solutions that result from these equations are the classical puddle solutions, for which the curvature increases linearly with depth. For explicit forms we refer to Landau \& Lifshitz (1959) and De Gennes, Brochard-Wyart \& Quéré (2013).

\subsubsection{Mismatch with outer solution 1}

Importantly, the puddle solution exhibits a finite curvature at the droplet's edge, namely $\kappa=2^{1 / 2} / \lambda_{c}$. This value of the curvature is obtained since, by symmetry, the puddle approaches the neck region vertically. This is to be contrasted with the curvature in the other outer region, below the drop, for which the curvature was 


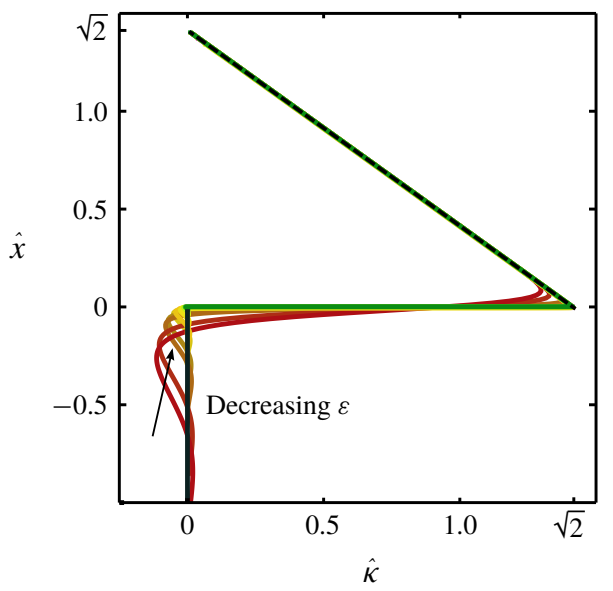

FIGURE 6. (Colour online) The interface curvature $\kappa$ in the neck region, obtained numerically from (3.15) for various evaporation rates $\mathcal{E}$. A distinctive jump in curvature can be seen, indicating a sharp pressure jump through the thin neck. The dashed line shows the puddle solution for outer region 2 .

found to be $1 / R$. For large drops, we thus find a 'mismatch' in curvature near the exit of the vapour layer. This implies that the problem requires an inner zone that smoothly joins the two outer regions. The matching is illustrated in figure 6 , where we present a numerical solution to the problem (details are given in the paragraphs below). The centre panel provides a detailed view of the droplet and pool profiles, and reveals a thin neck that is connected to the outer vapour layer. The curvature of these profiles are presented in figure 6 , for various values of $\mathcal{E}$. The dashed line corresponds to the puddle solution; the numerical profiles smoothly join the puddle to the vapour film of vanishing curvature at $\hat{x}=0$.

\subsection{Inner region: the neck profile}

\subsubsection{Matching conditions and numerical solution}

By inspection of figure 6 , the thin neck region represents a small vertical zone around $\theta=\pi / 2$. We therefore adopt a local Cartesian coordinate system, as sketched in figure 4, where gravity acts along the $x$-axis towards $x<0$. In this coordinate frame, we describe the drop interface as $d(x)$ and the pool interface as $p(x)$. The gap thickness is then expressed as $t(x)=d(x)-p(x)$ and its curvature $\kappa_{t}(x)=\kappa_{d}(x)-\kappa_{p}(x)$. The expressions for the pressure in the film from (2.1) and (2.2), for equal liquid properties and $k=0$, can be written as

$$
\left.\begin{array}{c}
P_{v}=-\rho g x-\gamma \kappa_{d} \\
P_{v}=-\rho g x+\gamma \kappa_{p} .
\end{array}\right\}
$$

Taking the difference of these equations, one finds $\kappa_{d}=-\kappa_{p}=\kappa_{t} / 2$, resulting in a symmetric deformation for both $d$ and $p$. Equation (2.8) then becomes

$$
\frac{1}{12 \eta_{v}} \partial_{x}\left[t^{3} \partial_{x}\left[\rho g x+\frac{1}{2} \gamma \partial_{x x} t\right]\right]=\frac{\epsilon}{t} \approx 0,
$$


where we used the small slope representation of the curvature $\kappa_{t}=t^{\prime \prime}$ for consistency with the lubrication approximation. Since we expect the local vapour generation in the small inner region to be negligible compared to the total generated flux, we can also drop the right-hand side. Since we expect the total generated flux to mainly come from the outer region 1 (verifiable a posteriori), we can neglect the flux contribution from the inter region and hence drop the right-hand side in the framework of the leading-order approximation. Ultimately the gap profile needs to be matched to that of the puddle shape $d(x)$. It is therefore convenient to express the lubrication profile by $d(x)=t(x) / 2$, and non-dimensionalise all lengths by $\lambda_{c}$ :

$$
\hat{d}=\frac{d}{\lambda_{c}}=\frac{t}{2 \lambda_{c}}, \quad \hat{x}=\frac{x}{\lambda_{c}} .
$$

With this, we obtain after integrating (3.13) once:

$$
\hat{d}^{\prime \prime \prime}=\frac{c^{3}}{\hat{d}^{3}}-1,
$$

where $c$ is an integration constant. This equation describes the shape of the drop interface inside the neck region, which is also found in the drainage problem, where $c$ is changing with time as the film thins.

The problem is closed by the matching conditions to the outer regions 1 and 2, respectively corresponding to negative and positive limits of $\hat{x}$. The curvature of outer region 1 scales as $1 / R$, which in dimensionless variables gives $\hat{d}^{\prime \prime} \sim 1 / \mathcal{R}$ and is thus asymptotically small for large drops, implying $\hat{d}^{\prime}(-\infty)=\hat{d}^{\prime \prime}(-\infty)=0$. Therefore, we require that $\hat{d}(-\infty)$ approaches a constant value, which according to (3.15) can be equated to $c$. This thickness must ultimately match the 'exit' thickness of outer zone 1 , leading to the condition

$$
\frac{t_{\text {exit }}}{2}=d(-\infty) \Longrightarrow c=\frac{1}{2} \cdot 1.22386 \cdots \mathcal{E}^{1 / 4} .
$$

The boundary condition for positive $\hat{x}$ comes from matching the curvature of the puddle solution, which in scaled units reads $\hat{d}^{\prime \prime}=\sqrt{2}$ (see $\$ 3.2$ ). We numerically solved (3.15) subject to these boundary conditions.

The resulting neck profiles are presented figure 7 for different values of $\mathcal{E}$, corresponding to different gap thickness according to (3.16). One can observe that reducing the flux $\mathcal{E}$ leads to a localisation of the neck region, both in terms of thickness and lateral extent. To highlight these trends we have reported the profiles on two panels with double logarithmic scales, centred around the position $\hat{x}_{n}$ of the minimum neck thickness (see caption for details). The same data were used to show the mismatch in curvature in figure 6. It is interesting to note that the profiles are strongly reminiscent of dimple profiles observed in dip coating (Snoeijer et al. 2008), which are indeed governed by an equation similar to (3.15). Apart from the dimple, the dip-coating solutions also exhibit the oscillations seen in figure 7(a), which were analysed in detail by Benilov et al. (2010).

\subsubsection{Self-similar solution for the neck region}

Based on our numerical results we observe that the neck region near position $\hat{x}_{n}$ becomes increasingly localised for small $\mathcal{E}$, while the neck thickness $\hat{d}_{n}$ is found to 

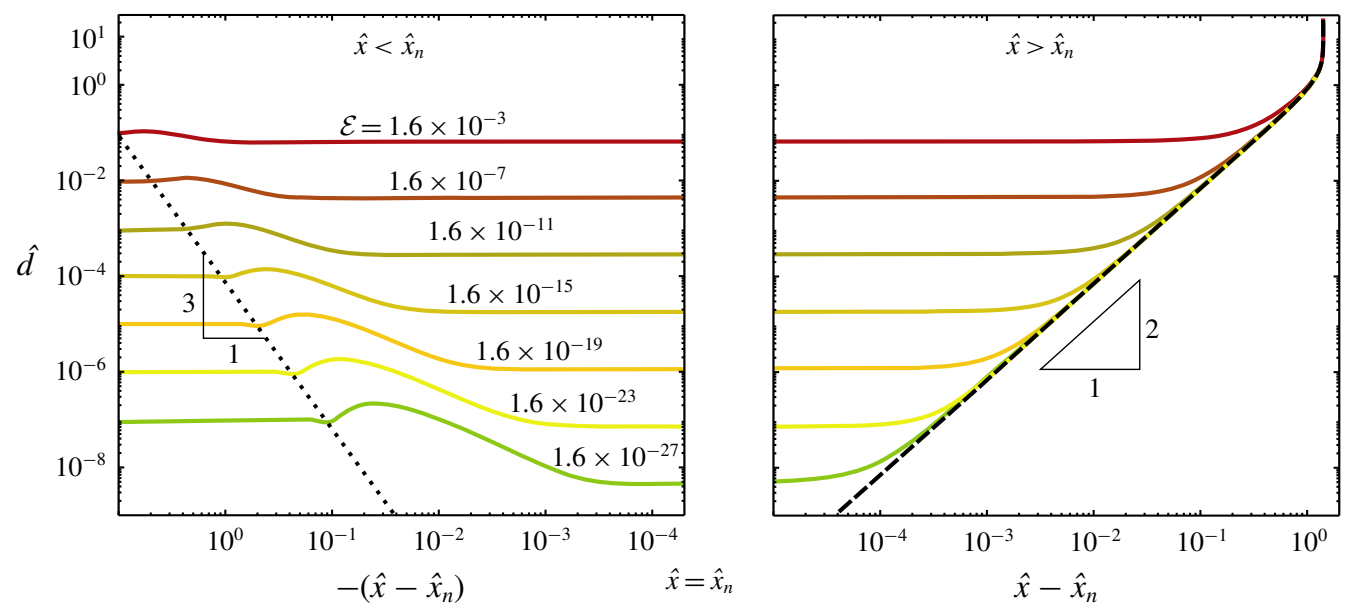

FIGURE 7. (Colour online) Neck profiles obtained from numerical solution of (3.15) for various vapour-layer thicknesses $\hat{d}(-\infty) \sim \mathcal{E}^{1 / 4}$ for the case of equal drop and pool properties and large drops $\left(R \gg \lambda_{c}\right)$. The double logarithmic representation of the two panels, with an inverse log-scale centred around the neck position $\hat{x}=\hat{x}_{n}$, reveals the details of the thin neck region and the oscillations upon approaching the vapour film. The dotted line indicates the location of the oscillations, scaling as $\hat{d} \sim\left(\hat{x}_{n}-\hat{x}\right)^{3}$. The dashed line shows the puddle solution for outer region 2 , exhibiting a $\hat{d} \sim\left(\hat{x}-\hat{x}_{n}\right)^{2}$ upon approaching the neck.

decrease. This is in direct analogy to the neck region for both settling drops and normal Leidenfrost drops above a rigid surface (Yiantsios \& Davis 1990; Snoeijer et al. 2009; Sobac et al. 2014). Owing to the smallness of $\hat{d}$ inside this region, (3.15) reduces to

$$
\hat{d}^{3} \hat{d}^{\prime \prime \prime}=c^{3},
$$

which means that the gravity is subdominant with respect to viscosity and surface tension. Indeed, equation (3.17) is identical to the neck equation studied, first by Wilson \& Jones (1983) in the context of film draining and later by Snoeijer et al. (2009) in the case of Leidenfrost drops, which admits similarity solutions

$$
\hat{d}(x)=c^{\alpha} T(\zeta), \quad \text { with } \zeta=\frac{\hat{x}-\hat{x}_{n}}{c^{\beta}} .
$$

Inserting this ansatz into (3.17) gives

$$
T^{3} T^{\prime \prime \prime}=1 \quad \text { and } \quad 4 \alpha-3 \beta=3 .
$$

The exponents $\alpha, \beta$ can be determined from a matching condition for $\zeta \gg 1$, for which the shape of the pool $\hat{d}(x) \simeq\left(\hat{x}-\hat{x}_{n}\right)^{2} / \sqrt{2}$ must be approached, regardless of the value of $c$. This implies for large $\zeta$,

$$
c^{\alpha} T(\zeta) \simeq c^{\alpha} \zeta^{2} / \sqrt{2}=c^{\alpha-2 \beta}\left(\hat{x}-\hat{x}_{n}\right)^{2} / \sqrt{2},
$$

hence, $\alpha-2 \beta=0$. Combined with (3.19), this gives the exponents $\alpha=6 / 5$ and $\beta=3 / 5$. 

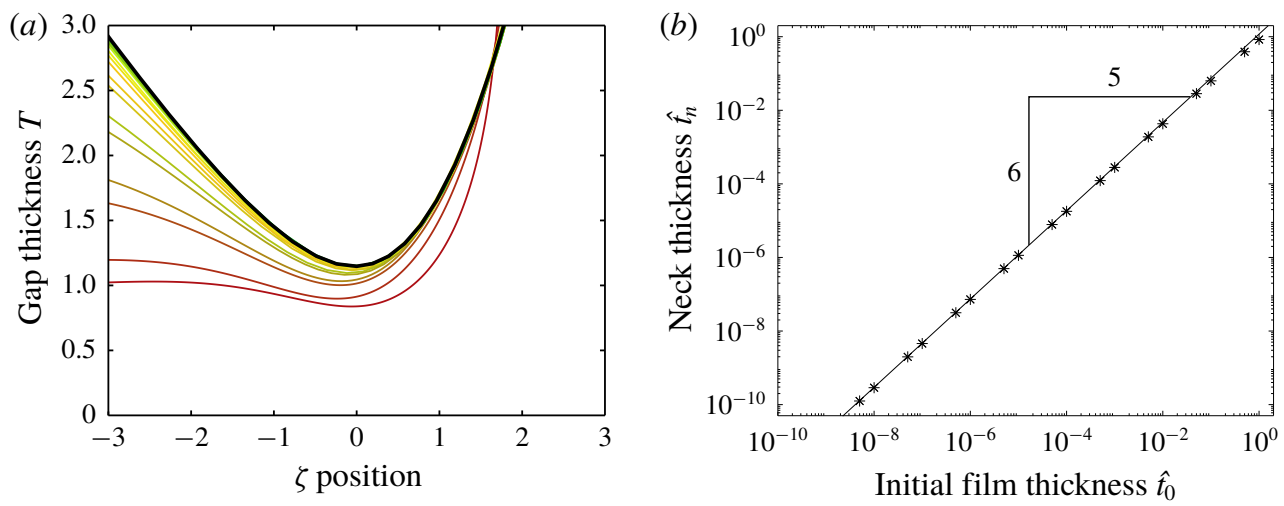

FIgURE 8. (Colour online) The neck region exhibits a self-similar structure, captured by the similarity function $T(\zeta)$ given by the dashed line $(a)$. The other curves represent numerical profiles of the neck shown in figure 7 for different $c$, scaled according to $T=\hat{d} / c^{6 / 5}$ and $\zeta=\left(\hat{x}-\hat{x}_{n}\right) / c^{3 / 5}$. Each of these profiles corresponds to a dot in $(b)$, where the straight solid line corresponds to the similarity law (3.21). The case of equal drop and pool properties and large drops $\left(R \gg \lambda_{c}\right)$.

While for large positive $\zeta$ we can impose the asymptotic boundary condition $T^{\prime \prime} \simeq$ $\sqrt{2}$, we still need to provide the asymptotics for negative $\zeta$. We now show that the matching to the film region implies $T^{\prime \prime} \simeq 0$ as the missing boundary condition. The matching to the film can in principle be obtained from a detailed analysis of the oscillatory approach to the thin film, in the spirit of the work on dip coating (Wilson \& Jones 1983; Benilov et al. 2010). Here we focus only on the first oscillation, which is sufficient for the present purpose. The typical slope of the neck solution $\hat{d}^{\prime} \sim c^{3 / 5} \sim$ $\mathcal{E}^{3 / 20}$ must be compared to that of the first bump. This bump has its own thickness scale $\hat{\delta}_{b}$ and lateral scale $\hat{\ell}_{b}$, such that we demand $\hat{\delta}_{b} / \hat{\ell}_{b} \sim \mathcal{E}^{3 / 20}$. For the approach of the bump we argue that all terms in (3.15) are involved, such that $\hat{d}^{\prime \prime \prime}$ must be of order unity, or $\hat{\delta}_{b} / \hat{\ell}_{b}^{3} \sim \mathcal{E}^{0}$. This scaling indeed gives the correct estimation for the position of the first oscillations in figure $7(a)$ (dotted line). Combining these two equations on $\hat{\delta}_{b}$ and $\hat{\ell}_{b}$ we find $\hat{\delta}_{b} \sim \mathcal{E}^{9 / 40}$ and $\hat{\ell}_{b} \sim \mathcal{E}^{3 / 40}$. The final step is to evaluate the curvature of the bump $\hat{\delta}_{b} / \hat{\ell}_{b}^{2} \sim \mathcal{E}^{3 / 40}$, which as anticipated, vanishes for small $\mathcal{E}$.

In summary, we expect the neck to be governed by a similarity solution $T(\zeta)$, which can be computed from (3.19) subject to boundary conditions $T^{\prime \prime}(-\infty)=0$ and $T^{\prime \prime}(\infty)=\sqrt{2}$. The numerical solution is given in figure 8 , represented as a dashed line. The other curves correspond to the profiles of figure 7, scaled according to (3.18). We observe a collapse onto the similarity solution as the value of $c \sim \mathcal{E}^{1 / 4}$ is reduced. The relation for the minimum neck thickness can now be found by determining the minimum of the similarity function, which we numerically find to be $T_{n}=1.147 \ldots$. Hence, we find

$$
\hat{d}_{n}=1.147 \cdots c^{6 / 5},
$$

which provides the minimum thickness at the neck, as confirmed in figure $8(b)$.

\subsection{Summary}

Let us now conclude the analysis for $\mathcal{E} \ll 1, \mathcal{R} \gg 1$ for the case where the drop and the pool consist of the same liquid $(\Gamma=1, \mathcal{P}=1)$. We first recall the expressions for 
the vapour-layer thickness below the centre of the drop $\left(t_{0}\right)$ and the vapour thickness as it approaches the neck $\left(t_{\text {exit }}\right)$ :

$$
t_{0}=\lambda_{c} \mathcal{E}^{1 / 4}, \quad t_{\text {exit }}=1.22386 \cdots \lambda_{c} \mathcal{E}^{1 / 4} .
$$

These can now be complemented by the minimum thickness of the neck

$$
t_{n}=2 \lambda_{c} \hat{d}_{n}=1.272 \cdots \lambda_{c} \mathcal{E}^{3 / 10},
$$

which was obtained using (3.16) and (3.21). The hierarchy of scales (2.13) is indeed satisfied and the approach is self-consistent as long as

$$
\mathcal{E}^{3 / 10} \ll \mathcal{E}^{1 / 4} \ll 1 \ll \mathcal{R} \ll \mathcal{E}^{-1 / 4},
$$

which completes the analysis.

\subsection{Hickman's boules}

We now briefly discuss the original boules of Hickman, where the vapour is generated from the superheated pool. As anticipated in $\S 2$, the vapour generation can be described by Newton's law of cooling: $j=\hbar \Delta T /\left(L \rho_{v}\right)$, where $\hbar$ is the heat transfer coefficient and the temperature difference now defined based on the (superheated) pool temperature far away from the drop (Bejan 1993). In this case, $j$ is approximately constant along the gap. Therefore, proceeding in a similar manner as discussed before in $\S 3$ we now obtain for the vapour thickness in outer region 1:

$$
\frac{1}{2 \sin \theta} \partial_{\theta}\left[\sin ^{2} \theta t^{3}\right]=\frac{6 j \eta_{v} R}{\rho g} .
$$

This is the equivalent of (3.4), now adapted to the Hickman boule. Solving this equation yields

$$
t(\theta)=\left(2 \frac{1-\cos \theta}{\sin ^{2} \theta}\right)^{1 / 3}\left(\frac{6 j \eta_{v} R}{\rho g}\right)^{1 / 3} .
$$

From this we deduce the (non-dimensional) vapour-layer thickness below the centre of the drop $\left(\tilde{t}_{0}=\tilde{t}(0)\right)$ and the vapour thickness as it approaches the neck $\left(\tilde{t}_{\text {exit }}=\tilde{t}(\pi / 2)\right)$ :

$$
\tilde{t}(0)=\left(\frac{6 j \eta_{v} R}{\rho g \lambda_{c}^{3}}\right)^{1 / 3}, \quad \tilde{t}(\pi / 2)=1.25992 \ldots\left(\frac{6 j \eta_{v} R}{\rho g \lambda_{c}^{3}}\right)^{1 / 3} .
$$

An important consequence of this result is that the thickness $t$ scales as $\Delta T^{1 / 3}$, which is fundamentally different from the $\Delta T^{1 / 4}$ scaling found previously. This new scaling law caries through to the thickness of the neck, according to $t_{\text {neck }} \sim t_{0}{ }^{6 / 5} \sim$ $\Delta T^{2 / 5}$, see $\S 3.3 .2$.

\section{Finite drop sizes and differing liquids}

Until now we have studied the structure of infinitely large Leidenfrost drops on a liquid bath of equal physical properties. It is of course interesting to extend the results to smaller-sized drops and to systems of different liquids. In the limit of small evaporation, $\mathcal{E} \ll 1$, one still finds that the vapour layer is asymptotically thin. Hence, the global shape of the drop is expected to be a 'superhydrophobic' drop on a pool, governed by hydrostatics. Exploiting this idea, we demonstrate that the various scaling laws for the vapour layer are robust, as is confirmed by solving the full problem numerically. 


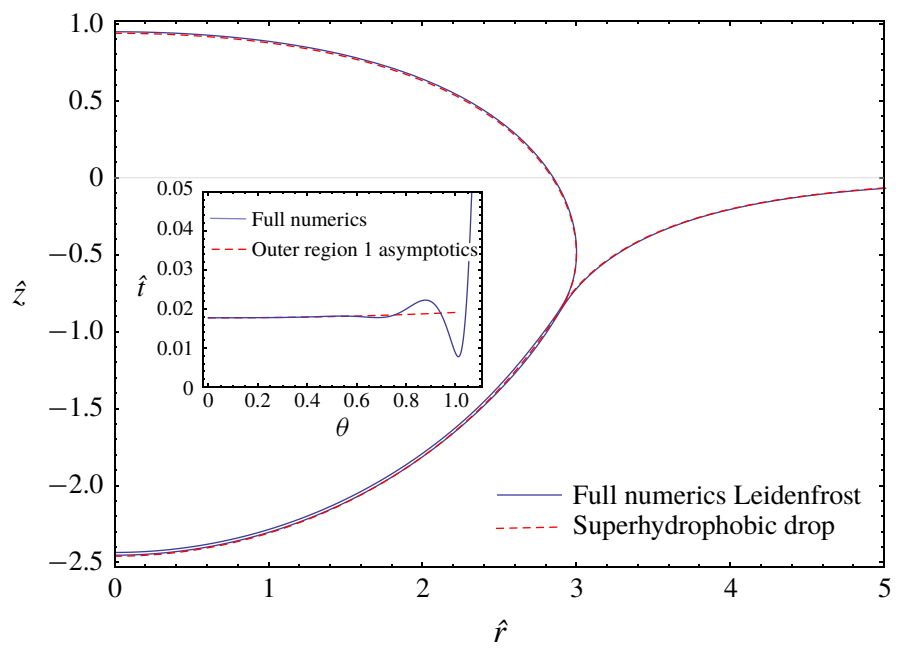

FIgURE 9. (Colour online) Profiles calculated for equal property liquids, $\mathcal{R}=3$ and $\mathcal{E}=$ $8.64 \times 10^{-8}$. Both the superhydrophobic drop calculation and the numerical simulation of the full problem yield a spherical cap solution of curvature $2 / \mathcal{R}_{c}$ for the gap geometry, in agreement with (3.2). Note that for finite-sized drop $\mathcal{R}_{c} \neq \mathcal{R}$ and the neck is positioned at $\theta_{n}=1.01$.

\subsection{Finite drop size}

Let us first focus on finite-sized drops, while keeping $\Gamma=\mathcal{P}=1$. The size of the drop can be tuned by the value of $k$ appearing in (2.2), and a numerical example is presented in blue in figure 9. In this particular case the droplet radius $\mathcal{R}=R / \lambda_{c}=$ 3 (as seen from above); in general, a relation $\mathcal{R}(k)$ can be established numerically (cf. appendix A). Comparing the droplet shape to that of the very large drops in figure 3, one finds that the immersed part of the drop still resembles a spherical cap, but the position of the neck has clearly shifted, resulting in the fact that drop radius $\mathcal{R}$ is now smaller than the (dimensionless) radius of curvature of the spherical cap, which we define as $\mathcal{R}_{c}$. The inset shows details of the vapour layer, which also has a similar structure as compared to large drops at small $\mathcal{E}$.

These features can be understood in detail. First, we compare the full numerical solution to the reduced (hydrostatic) calculation for the superhydrophobic drop, as described in $\S 2.2$ (and in more detail in appendix B). The latter is shown as the red dashed curve in figure 9, indeed giving an excellent description of the global shape. As a second step, one can use this global shape to predict the gap thickness. Namely, the superhydrophobic drop provides $P_{v}$ assuming a negligible back influence of the vapour film profile on $P_{v}$; this is valid except for the relatively narrow neck and intermediate regions where the capillary (Laplace) pressure due to vapour film deformation is important. Inserting this pressure profile into the lubrication equation (2.8), one can obtain the vapour-layer profile. The result is shown as the red dashed curve in the inset of figure 9 and indeed manifests an excellent quantitative agreement, outside the neck region and in the oscillatory intermediate region.

The same asymptotic analysis as for the infinite drop can be applied. However, care must be taken that the oscillation visible in the inset of figure 9 does not extend all the way to the centre of the drop; otherwise there would be no flat 'outer region' below the drop. Hence, we need the width of the bump, $\hat{\ell}_{b} \sim \mathcal{E}^{3 / 40}$ to remain smaller 
than $\mathcal{R}$. We therefore postulate a new hierarchy of length scales in the case of finite drop sizes, namely

$$
t_{n} \ll t_{0} \ll \ell_{b} \ll R,
$$

which is the same as $\hat{t}_{n} \ll \hat{t}_{0} \ll \hat{\ell}_{b} \ll \mathcal{R}$, resulting in

$$
\mathcal{E}^{3 / 10} \ll \mathcal{E}^{1 / 4} \ll \mathcal{E}^{3 / 40} \ll \mathcal{R}
$$

Based on these observations we can now revisit the analysis for the vapour layer. For equal material properties and small evaporation numbers, equation (3.2) is still valid so that the immersed part of the drop has a constant curvature. For the numerical example in figure 9 we find $\lambda_{c} \kappa_{h}=2 \times 0.29=2 / \mathcal{R}_{c}$. Also, the lubrication equation (3.7) is still valid. However, since the expression for $P_{v}$ involves $\mathcal{R}_{c}$ rather than $\mathcal{R}$, we need to adapt the expression for $t_{*}=t_{0}$ accordingly. With this, equation (3.5) and the first expression (3.10) simply become

$$
t_{*}=t_{0}=\lambda_{c}\left(\frac{\mathcal{R}_{c}}{\mathcal{R}}\right)^{1 / 4} \mathcal{E}^{1 / 4},
$$

where the ratio $\mathcal{R}_{c} / \mathcal{R}$ can be calculated from the corresponding superhydrophobic drops. When computing the thickness of the very thin neck, i.e. the position where the drop and pool are the closest, one should take into account two further effects due to the finite drop size. First, the neck is no longer positioned at $\theta=\pi / 2$ : in the example in figure 9 we find $\theta_{n}=1.01$, which will lead to a small change in the thickness at the 'exit' of the outer region, $t_{\text {exit }}$, in view of the weak variation of $t$ with theta. Second, the matching of the neck to the upper surface of the droplet will be modified, since the droplet's curvature will change with respect to the value for an infinitely large drop. Since all these factors have to be evaluated numerically, we here just give the scaling of the neck thickness

$$
t_{n} \sim \lambda_{c}\left(\frac{\mathcal{R}_{c}}{\mathcal{R}}\right)^{3 / 10} \mathcal{E}^{3 / 10} .
$$

We also note that the prefactor in this law exhibits some dependence on $\mathcal{R}$ when $\mathcal{R} \approx 1$, and therefore the scaling $\hat{t}_{n} \sim \mathcal{R}^{3 / 10}$ may not actually hold for $\mathcal{R} \approx 1$. This explains why the apparent scaling identified by Maquet et al. (2016) was rather $\hat{t}_{n} \sim$ $\mathcal{R}^{1 / 4}$ for the range of radii studied there.

In summary, we conclude that the structure of the present asymptotic analysis and the resulting scaling laws remain the same for finite-sized drops, provided that the undulations near the neck do not penetrate a large fraction of the gap length (i.e. provided that the intermediate region stays significantly shorter than the outer region 1). This being satisfied, in the case of equal liquid properties one can even compute the prefactors, provided that $\mathcal{R}_{c}$ and $\theta_{n}$ are determined by considering the corresponding superhydrophobic drop. Note that the (dimensional) drop size $R$ appears both in $\mathcal{E}$ and in the prefactors of (4.3) and (4.4). Hence, as already mentioned, one observes a pure scaling relation in terms of $R$ only in the large drop limit, although this is limited to the case of equal liquid properties.

We close the discussion by considering the case when $\Gamma=\gamma_{p} / \gamma_{d} \neq 1$ and $P=\rho_{p} / \rho_{d} \neq 1$, i.e. when the drop and pool consist of different liquids. This is for example the case for the ethanol drops on a silicone oil pool studied by Maquet et al. (2016). Note that the immersed shape is now no longer expected to 
be a spherical cap due to the generally different densities of the two liquids. As a consequence, we will no longer be able to proceed in fully analytical terms as before. However, asymptotic analyses can still be carried out if we rely on the numerical solution for the equilibrium (static) shape of the associated superhydrophobic drop, which is expected to constitute the leading-order approximation for the Leidenfrost drop shape. (That is possessing the same size $R$ and liquid properties as our Leidenfrost drop, the liquid-liquid interfacial tension being equal to $\gamma_{d}+\gamma_{p}$.) The scaling laws $t_{0} \sim \lambda_{c} \tilde{\mathcal{E}}^{1 / 4}$ and $t_{n} \sim \lambda_{c} \tilde{\mathcal{E}}^{3 / 10}$ will eventually be recovered, the prefactors being determined numerically through the associated superhydrophobic drop characteristics. We formalise these points below.

We use $\lambda_{c}$ as the length scale and $\rho_{d} g \lambda_{c}=\gamma_{d} / \lambda_{c}$ as the pressure scale to introduce dimensionless variables, marked by a hat,

$$
\hat{s}=\frac{s}{\lambda_{c}}, \quad \hat{r}=\frac{r}{\lambda_{c}}, \quad \hat{z}=\frac{z}{\lambda_{c}}, \quad \hat{P}_{v}=\frac{P_{v}}{\rho_{d} g \lambda_{c}},
$$

similar to earlier used $\hat{t}=t / \lambda_{c}$. Here $r$ is the cylindrical radial coordinate (distance to the symmetry axis), $s$ the arc length (counting from the symmetry axis) along the liquid-liquid interface of the associated superhydrophobic drop in the meridional crosssection, while $z$ is the vertical Cartesian coordinate (cf. figure 3). In the outer region 1 considered in the framework of our present asymptotic scheme (cf. figure 3 and $\S 3$ ), equation (2.8) for the vapour gap thickness then rewrites to leading order as

$$
-\frac{1}{12 \hat{r}} \partial_{\hat{s}}\left(\hat{r} \hat{t}^{3} \partial_{\hat{s}} \hat{P}_{v}\right)=\frac{\tilde{\mathcal{E}}}{\hat{t}},
$$

where the pressure in the vapour gap $\hat{P}_{v}(\hat{s})$ and the geometric characteristics $\hat{r}(\hat{s})$ are a priori given functions, known from the associated superhydrophobic drop consideration (cf. appendix B). The solution to (4.6), non-singular at the symmetry axis $\hat{s}=0$, can be written as

$$
\hat{t}^{4}=\frac{16 \tilde{\mathcal{E}} \int_{0}^{\hat{s}} \hat{r}^{4 / 3}\left(-\hat{P}_{v}^{\prime}\right)^{1 / 3} \mathrm{~d} \hat{s}}{\left(-\hat{r} \hat{P}_{v}^{\prime}\right)^{4 / 3}}
$$

the prime denoting a derivative with respect to $\hat{s}$. The thickness at the symmetry axis, $\hat{t}_{0}=\hat{t}(0)$, and at the exit, $\hat{t}_{\text {exit }}=\hat{t}\left(\hat{s}_{C L}\right)$, can then be inferred as

$$
\hat{t}_{0}=\left(-\frac{6 \tilde{\mathcal{E}}}{\hat{P}_{v}^{\prime \prime}(0)}\right)^{1 / 4}, \quad \hat{t}_{\text {exit }}=\frac{2 \tilde{\mathcal{E}}^{1 / 4}\left(\int_{0}^{\hat{s}_{C L}} \hat{r}^{4 / 3}\left(-\hat{P}_{v}^{\prime}\right)^{1 / 3} \mathrm{~d} \hat{s}\right)^{1 / 4}}{\left[-\hat{r}\left(\hat{s}_{C L}\right) \hat{P}_{v}^{\prime}\left(\hat{s}_{C L}\right)\right]^{1 / 3}},
$$

where $\hat{s}_{C L}$ is the value of the arc length at the contact line of the associated superhydrophobic drop (known a priori, cf. appendix B). We note that the consideration only makes sense provided that $\hat{P}_{v}^{\prime}<0$ for all $0<\hat{s}<\hat{s}_{C L}$ and $\hat{P}_{v}^{\prime \prime}(0)<0$, which turns out to be indeed the case for a superhydrophobic drop. We also note that the results of $\S 3$ with a hemispherical shape and of $\S 4.1$ with a spherical cap shape are recovered from here with $\mathrm{d} \hat{s}=\mathcal{R} \mathrm{d} \theta, \hat{P}_{v}^{\prime}=-\sin \theta, \hat{r}=\mathcal{R} \sin \theta$ and $\hat{s}_{C L}=\mathcal{R} \theta_{C L}$. Equations (4.7) and (4.8) confirm once again that the scaling in terms of $\mathcal{E}$ established in $\S 3$ for the 
vapour gap thickness in this outer region 1 , namely $\hat{t}=O\left(\tilde{\mathcal{E}}^{1 / 4}\right)$ including $\hat{t}_{0}=O\left(\tilde{\mathcal{E}}^{1 / 4}\right)$ and $\hat{t}_{\text {exit }}=O\left(\tilde{\mathcal{E}}^{1 / 4}\right)$, is indeed robust.

We next turn to the inner region (cf. figure 3), also referred to as the neck region, the consideration of which follows the same asymptotic scheme as $\S 3$. We introduce a local Cartesian coordinate $x$ (and its dimensionless version $\hat{x}=x / \lambda_{c}$ ) parallel to the slope at the contact line of the associated superhydrophobic drop and pointing away from the vapour film. In this local Cartesian system, the drop and pool surfaces are described by $\hat{d}(\hat{x})$ and $\hat{p}(\hat{x})$, respectively, and $\hat{t}=\hat{d}-\hat{p}$ and $\mathrm{d} \hat{x} \approx \mathrm{d} \hat{s}$. Similar to $\S 3$, the vapour pressure $\hat{P}_{v}$ in the neck region (unlike the earlier considered outer region 1 ) is no longer given by the associated superhydrophobic drop but is rather coupled to the local vapour gap profile. Owing to the expected small size of the neck region, the leading-order contribution in $\partial_{\hat{x}} \hat{P}_{v}$ will be due to the capillary (Laplace) pressure associated with the first curvature of the drop and pool surfaces, i.e. $\hat{P}_{v}^{\prime} \approx-\hat{d}^{\prime \prime \prime}$ and $\hat{P}_{v}^{\prime} \approx \Gamma \hat{p}^{\prime \prime \prime}$. The latter two expressions must be equal, hence $\hat{d}^{\prime \prime \prime} \approx-\Gamma \hat{p}^{\prime \prime \prime}$. Using this fact, as well as $\hat{t}=\hat{d}-\hat{p}$, we express $\hat{P}_{v}^{\prime} \approx-(\Gamma /(1+\Gamma)) \hat{t}^{\prime \prime \prime}$. Using this in (4.6) and recalling, on the one hand, that it is planar geometry that holds to leading order in the neck region, and on the other hand, that the local evaporation flux $\tilde{E} / \hat{t}$ is negligible relative to the flux from the remainder of the vapour gap passing through the neck region (cf. §3.3), one obtains

$$
\frac{1}{12} \frac{\Gamma}{1+\Gamma} \partial_{\hat{x}}\left(\hat{t}^{3} \partial_{\hat{x} \hat{x} \hat{x}} \hat{t}\right)=0,
$$

where the prefactor is kept for later convenience.

As in $\$ 3.3$, we shall look for solutions of this equation subject to boundary conditions $\hat{t}^{\prime \prime}(-\infty)=0$ whilst $\hat{t}^{\prime \prime}(+\infty)=\hat{\kappa}_{1 h, C L}-\hat{\kappa}_{1 e, C L}$, where $\hat{\kappa}_{1 h, C L}$ and $\hat{\kappa}_{1 e, C L}$ are the (known) first curvatures of the upper drop and pool surfaces at the contact line of the associated superhydrophobic drop (appendix B). Recall that in the particular case of large drops with the same liquid properties (cf. $\S 3.3$ ), we have $\hat{\kappa}_{1 h, C L}=\sqrt{2}$ and $\hat{\kappa}_{1 e, C L}=-\sqrt{2}$ resulting from the puddle solution, and giving rise to $\hat{t}^{\prime \prime}(+\infty)=2 \sqrt{2}$ in present terms.

To account for the flux coming from the interior of the vapour gap, against which the local flux $\tilde{E} / \hat{t}$ was neglected when writing (4.9), we integrate (4.9) from $\hat{x}=-\infty$ to a finite value of $\hat{x}$. In doing so, we take into account that the quantity $\left.(1 / 12)(\Gamma /(1+\Gamma)) \hat{t}^{3} \partial_{\hat{x} \hat{x} \hat{x}} \hat{t}\right|_{\hat{x}=-\infty}$, which is actually the dimensionless volume flux per unit length at the entrance to the neck region, must match the corresponding flux from the outer region 1 (the contribution from the intermediate region being negligible to leading order similar to $\S 3$ ). Thus, it must be equal to the dimensionless volume flux per unit length at the exit from the outer region 1 :

$$
\frac{1}{\hat{r}\left(\hat{s}_{C L}\right)} \int_{0}^{\hat{s}_{C L}} \hat{r} \frac{\tilde{\mathcal{E}}}{\hat{t}} \mathrm{~d} \hat{s}=\frac{1}{12} \hat{t}_{\text {exit }}^{3}\left[-\hat{P}_{v}^{\prime}\left(\hat{s}_{C L}\right)\right],
$$

the latter equality being written on account of (4.6) integrated over the outer region 1. Here recall that $\hat{t}_{\text {exit }}$ is given by (4.8), while $\hat{r}\left(\hat{s}_{C L}\right)$ and $\hat{P}_{v}^{\prime}\left(\hat{s}_{C L}\right)$ are values known from the associated superhydrophobic drop consideration (appendix B). We thereby finally arrive at the following equation for the vapour gap thickness in the inner (neck) region:

$$
\hat{t}^{3} \hat{t}^{\prime \prime \prime}=\frac{1+\Gamma}{\Gamma} \hat{t}_{\text {exit }}^{3}\left[-\hat{P}_{v}^{\prime}\left(\hat{s}_{C L}\right)\right],
$$


which is a first integral of (4.9) accounting for the mentioned flux condition. A rescaling

$$
\hat{t}=A^{2 / 5} B^{-3 / 5} T, \quad \hat{x}=A^{1 / 5} B^{-4 / 5} \zeta, \quad A \equiv \frac{1+\Gamma}{\Gamma} \hat{t}_{e x i t}^{3}\left[-\hat{P}_{v}^{\prime}\left(\hat{s}_{C L}\right)\right], \quad B \equiv \frac{\hat{\kappa}_{1 h, C L}-\hat{\kappa}_{1 e, C L}}{\sqrt{2}}
$$

$(4.12 a-d)$

reduces equation (3.17) with the earlier mentioned boundary conditions to the problem $T^{3} T^{\prime \prime \prime}=1$ with $T^{\prime \prime}(-\infty)=0$ and $T^{\prime \prime}(+\infty)=\sqrt{2}$ already considered in $\S 3.3$. In particular, for the minimum neck thickness, $T_{n}=1.147$ was obtained, which here yields

$$
\hat{t}_{n}=1.147\left(\frac{1+\Gamma}{\Gamma} \hat{t}_{e x i t}^{3}\left[-\hat{P}_{v}^{\prime}\left(\hat{s}_{C L}\right)\right]\right)^{2 / 5}\left(\frac{\hat{\kappa}_{1 h, C L}-\hat{\kappa}_{1 e, C L}}{\sqrt{2}}\right)^{-3 / 5} .
$$

As $\hat{t}_{\text {exit }}=O\left(\tilde{\mathcal{E}}^{1 / 4}\right)$ in accordance with (4.8), whereas the other quantities in (4.13) are just $O(1)$, we see that $\hat{t}_{n}=O\left(\tilde{\mathcal{E}}^{3 / 10}\right)$, which confirms the robustness of the earlier established scaling law for the neck thickness (cf. §3).

With the $\tilde{\mathcal{E}}$ scaling (power) laws themselves confirmed, we recall that the prefactors at the asymptotic results such as (4.7), (4.8) and (4.13) depend exclusively on characteristics of the associated superhydrophobic drop. The latter are computed numerically as described in appendix B and subsequently used in (4.7), (4.8) and (4.13) to complete the present asymptotic consideration. The results are illustrated below together with their comparison with the full Leidenfrost numerics (the latter realised as described in appendix A).

Figure 10 shows results for a drop of $\mathcal{R}=3$ with non-equal material properties inspired from Maquet et al. (2016). We see that the global Leidenfrost drop and pool shapes are still close to those of the associated superhydrophobic drop, as expected. The morphological structure of the vapour gap is still the same as noted earlier in the case of equal material properties. The asymptotic results for the outer region 1 capture well the film thickness distribution in the central part, although at larger $\tilde{\mathcal{E}}$ the waviness from the intermediate region penetrates closer to the symmetry axis. For the thickness values in the centre and at the neck, we once again obtain a good agreement between the asymptotic and the full numerical approaches, although the agreement slightly deteriorates at larger $\tilde{\mathcal{E}}$ (especially for $\hat{t}_{n}$ ). Importantly, the asymptotic scalings $\hat{t}_{0} \sim \tilde{\mathcal{E}}^{1 / 4}$ and $\hat{t}_{n} \sim \tilde{\mathcal{E}}^{3 / 10}$ are seen to still be well reproduced by the full numerics. We note that the straight lines in figure $10(d)$ are the asymptotic predictions with no fitting involved: the prefactors were determined directly from the associated superhydrophobic drop analysis.

Some of the cases shown in figures 9 and 10 are taken up for a further parametric study in figures 11 and 12, partly aimed at testing the limits of the applicability of the present asymptotic scheme. In particular, the results of figure 10 corresponding to the larger evaporation number value $\left(\tilde{\mathcal{E}}=2.3 \times 10^{-7}\right)$ are extended in figure 11 to some other drop radius values. While the Leidenfrost drops are seen to still be close to the superhydrophobic shapes in all cases shown, the present asymptotic results cease to be valid for smaller drops as far as the outer region 1 and $\hat{t}_{0}$ are concerned. This is especially true for the smallest drop displayed, with $\mathcal{R}=1.5$. It can clearly be observed from figure 11(b) that such a change in the vapour gap morphology is related first of all with the violation of the part $\hat{l}_{n} \ll \mathcal{R}$ of the presumed hierarchy of length scales (4.1). This violation occurs for larger $\tilde{\mathcal{E}}$ as $\mathcal{R}$ is decreased, when the wavelength of the intermediate region undulations becomes comparable with the size of the drop, 
(a)

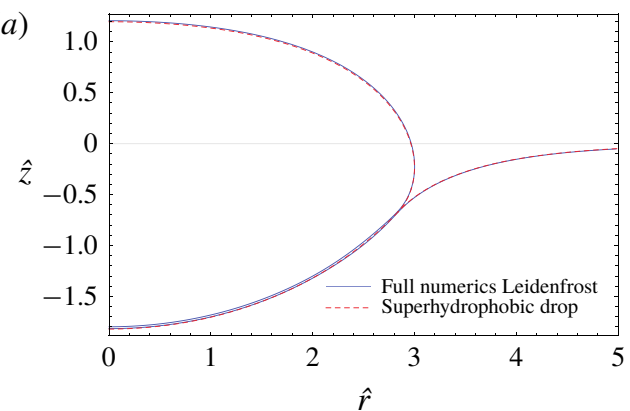

(c)

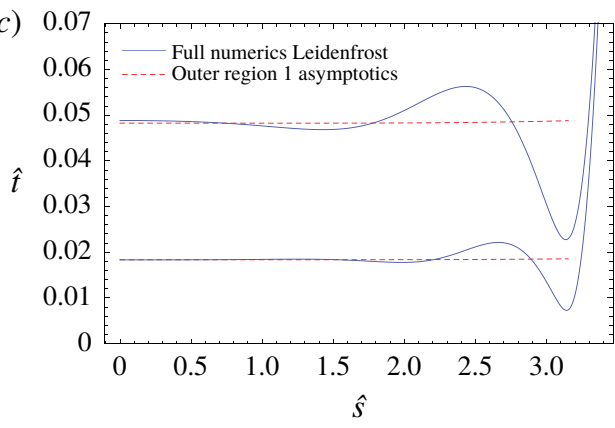

(b)



(d)

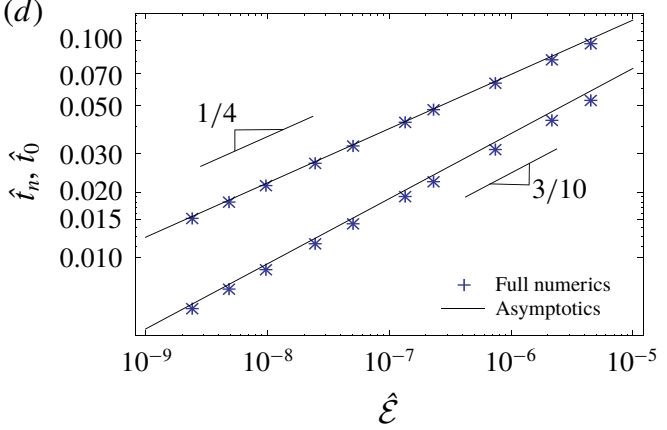

FIGURE 10. (Colour online) Global drop shapes $(a, b)$ and vapour gap thicknesses ( $c$ distributions along the arc length of the pool surface; $d$ values in the centre and at the neck as functions of $\tilde{\mathcal{E}}$ ). Results for $\mathcal{R}=3$ and the material properties resembling those for an ethanol drop on a silicone oil pool (Maquet et al. 2016). In particular, $\mathcal{P}=1.244$ and $\Gamma=1.156$. The $(a, b)$ are for $\tilde{\mathcal{E}}=4.8 \times 10^{-9}(\Delta T=1 \mathrm{~K})$ and $\tilde{\mathcal{E}}=2.3 \times 10^{-7}(\Delta T=40 \mathrm{~K})$, respectively. And so are the lower and upper curves in $(c)$. The arc length $\hat{s}$ is along the pool surface in the full numerics, while along the superhydrophobic drop-pool interface in the outer region 1 asymptotics.

as already discussed at the end of $\S 4.1$. At smaller $\tilde{\mathcal{E}}$ however (e.g. at $\tilde{\mathcal{E}}=4.8 \times 10^{-9}$ used earlier in figure 10), the present asymptotic scheme is still found to work rather satisfactorily in the central part of the drop even for $\mathcal{R}$ as low as $\mathcal{R}=1.5$ (the result not shown).

A no less remarkable result of figure 11 is some deterioration of the agreement between asymptotics and full numerics observed for large $\mathcal{R}$ (viz. $\mathcal{R}=10$ ) as compared to $\mathcal{R}=3$ as far as the gap thickness in the centre (i.e. $\hat{t}_{0}$ ) is concerned. There is a good reason for that. Indeed, it is clear that, quite unlike a superhydrophobic drop over a pool with equal liquid properties, a drop of a differing liquid will tend to adopt a puddle-like shape as $\mathcal{R}$ is increased (here limiting consideration to the case $\mathcal{P}>1$, of a lighter drop; a heavier one will merely sink for sufficiently large $\mathcal{R}$ ). For such a puddle, which the drop with $\mathcal{R}=10$ of figure 11 already much resembles, it is not only the upper surface that flattens, but also the immersed one. The present asymptotic scheme breaks down in the presence of a flattened part of the immersed surface, for which the $\hat{P}_{v}^{\prime}$ and $\hat{P}_{v}^{\prime \prime}$ values to be used in (4.7) and (4.8) vanish and the driving force of the flow $\hat{P}_{v}^{\prime}$ is no longer accurately estimated by the superhydrophobic drop. The length scale at which such flattening occurs is the (dimensionless) capillary 
(a)

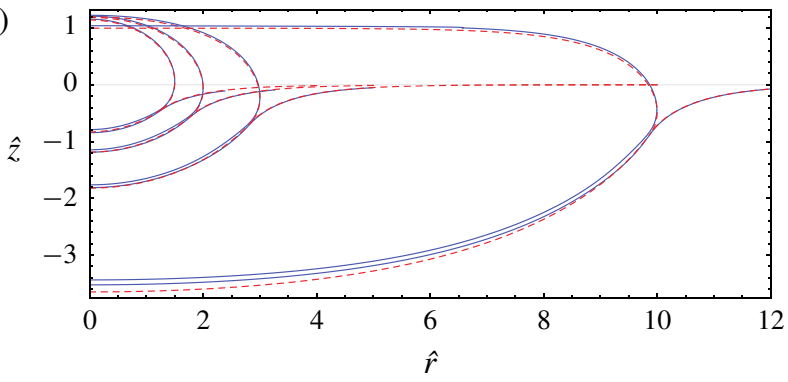

(b)

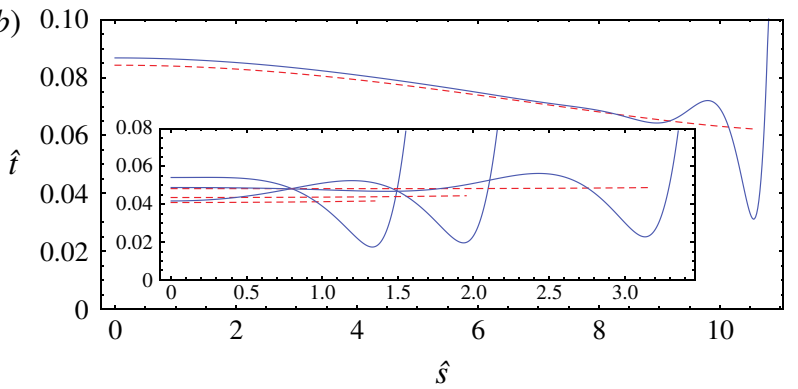

FIGURE 11. (Colour online) Extension of the part of the results of figure 10 pertaining to $\tilde{\mathcal{E}}=2.3 \times 10^{-7}$ (still with $\mathcal{P}=1.244, \Gamma=1.156$ ) to a number of drop radii, $\mathcal{R}=$ $1.5,2,3,10$. In $(b)$, the correspondence between each curve and the $\mathcal{R}$ value can reasonably be guessed from the right ending of the former.
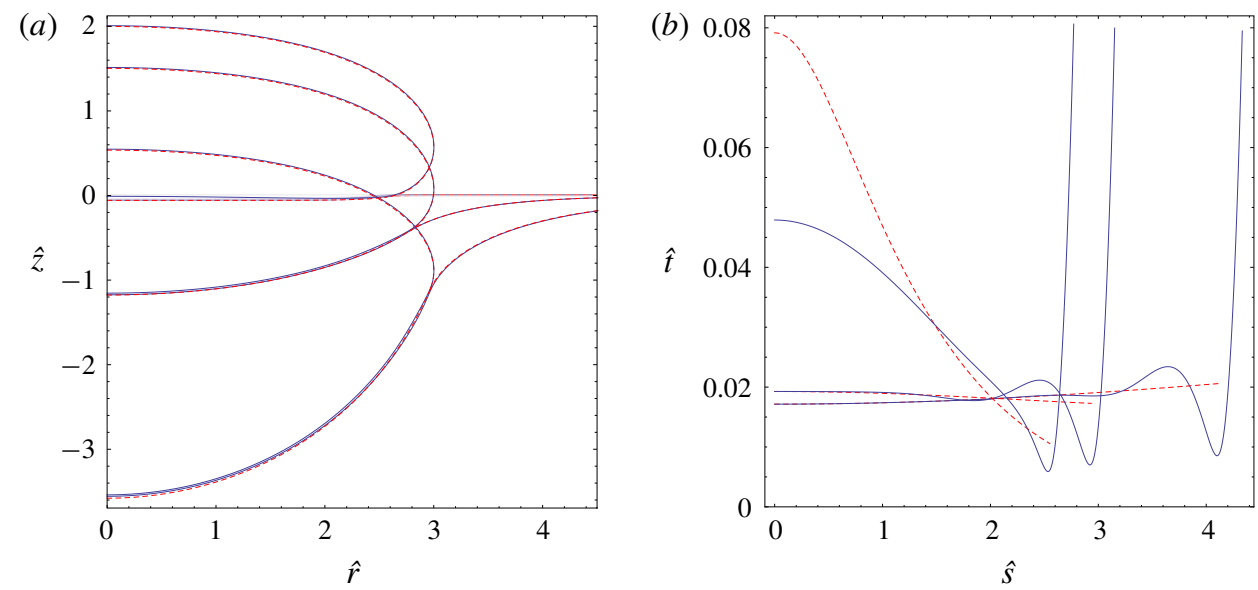

FIgURE 12. (Colour online) Extension of the results of figure 9 to a number of pool-todrop density ratios $\mathcal{P}=0.8,2,40$ (otherwise still for $\mathcal{R}=3, \Gamma=1$ and $\tilde{\mathcal{E}}=4.8 \times 10^{-9}$, which corresponds to $\mathcal{E}=8.64 \times 10^{-8}$ ). As $\mathcal{P}$ is increased, the drop mounts in $(a)$, while the curves in $(b)$ get shorter from the right (the overall arc length of the vapour gap decreases).

length of the immersed surface $\sqrt{(1+\Gamma) /(\mathcal{P}-1)}$. Hence the present asymptotic scheme is expected to work in an optimal way when $\mathcal{R} \lesssim \sqrt{(1+\Gamma) /(\mathcal{P}-1)}$, i.e. for sufficiently small drops. The drop with $\mathcal{R}=10$ of figure 11 is already relatively large 
in this regard, hence the mentioned agreement deterioration. Note that this concerns a relatively larger value $\tilde{\mathcal{E}}=2.3 \times 10^{-7}$. For a smaller value $\tilde{\mathcal{E}}=4.8 \times 10^{-9}$, the agreement still proves to be excellent even for $\mathcal{R}=10$ (the result not shown). Note also that for a system with $\mathcal{P}=1$, the capillary length of the immersed surface goes to infinity (the surface itself assuming the form of a spherical cap), and hence such a limitation on the maximum drop radius is lifted.

In figure 12 , we take up the case of figure 9 (with $\mathcal{R}=3, \Gamma=1$ and $\tilde{\mathcal{E}}=4.8 \times 10^{-9}$ ) and explore how the phenomenon is affected by the variation of the density ratio $\mathcal{P}$. We see that, as long as the pool surface deformation remains appreciable (e.g. for $\mathcal{P}=0.8,2$ ), the expected morphological features remain in place. In particular, this is still the case at $\mathcal{P}=0.8$, for nearly the maximum possible immersed-surface deformation: we are then on the verge of the disappearance of a floating drop configuration for $\mathcal{P}$ slightly below $\mathcal{P}=0.8$, when a drop with $\mathcal{R}=3$ and $\Gamma=1$ becomes too heavy to remain on the pool surface. The fact that such disappearance happens practically simultaneously (in terms of $\mathcal{P}$ ) for the Leidenfrost and superhydrophobic drops can serve as yet another confirmation of a close relationship between these two configurations. On the other hand, while perhaps not physically relevant, the interest of the case $\mathcal{P}=40$ is that the pool surface becomes essentially flat: hence we should thereby approach the case of a Leidenfrost drop above a (flat) solid. Indeed, the vapour film profile for $\mathcal{P}=40$ is seen (cf. figure 12b) to more closely resemble that typical for a flat solid substrate (Snoeijer et al. 2008; Burton et al. 2012; Sobac et al. 2014), with the present asymptotic scheme yielding an appreciable error with respect to the full numerics. The reason for such an error can be traced back to the fact that, under the conditions when the vapour gap thickness becomes comparable to (or even larger than) the deviation of the immersed surface from the horizontal, the pressure distribution $\hat{P}_{v}(\hat{S})$ can no longer be taken as $\hat{t}$-independent as assumed from the superhydrophobic drop considerations. There must rather be a feedback between $\hat{P}_{v}(\hat{s})$ and $\hat{t}(\hat{s})$ such as that which exists in the case of a flat solid substrate (Snoeijer et al. 2008; Sobac et al. 2014). Note also that a similar observation can actually be attributed to the flattened parts (if any) of the immersed surface even if the latter is not flattened overall (cf. the above discussion of the drop with $\mathcal{R}=10$ of figure 11). Finally, an increase of the pool-to-drop surface tension ratio $\Gamma$ will lead to similar effects of pool surface flattening as those due to an increase of $\mathcal{P}$.

Thus, for differing liquids too, the scalings $\hat{t}_{0} \sim \tilde{\mathcal{E}}^{1 / 4}$ and $\hat{t}_{n} \sim \tilde{\mathcal{E}}^{3 / 10}$ are still robust and work in a reasonable interval of small $\tilde{\mathcal{E}}$ values for sufficiently large drops $\left(\mathcal{R} \gg \hat{l}_{n}\right)$ in the general case of significantly curved pool surfaces. However, the particular cases when the immersed superhydrophobic drop surface undergoes a strong overall (for too large $\mathcal{P}$ and/or $\Gamma$ ) or partial (for too large $\mathcal{R}$ ) flattening must realistically be excluded from the applicability domain of the present asymptotic scheme. Indeed, such applicability would then just be expected for extremely small values of $\tilde{\mathcal{E}}$, which are of no practical interest. In contrast, the pure scaling in terms of $\mathcal{R}$ underscored in $\S 4.1$ makes actually no sense here (for differing liquids) due to a morphological difference between the superhydrophobic drop shapes in the limit of large $\mathcal{R}$ : a hemisphere for equal liquid properties, and a puddle for differing ones (assuming a lighter drop, $\mathcal{P}>1$ ) with further adverse consequences due to the mentioned partial flattening of the immersed surface. 


\section{Conclusion}

Leidenfrost drops on a superheated liquid pool were studied in the limit of small evaporation numbers $\tilde{\mathcal{E}}$, the latter proportional to the superheat $\Delta T$ and determined by both the thermal and hydrodynamic properties of the system. The pool surface being deformed under the drop, the vapour gap was found to be of quite a different morphology as compared to that of Leidenfrost drops deposited on a superheated flat plate. The reason is that, for a curved substrate, there exists an a priori given driving pressure gradient in the vapour layer, determined by the associated superhydrophobic drop configuration and independent to leading order of the gap thickness distribution. In contrast, for a flat substrate, the pressure gradient is fully determined by the variation of the gap thickness.

As shown in detail in figure 3, three main asymptotic regions are identified to describe the thin gap of vapour between the droplet and the liquid pool. This is unlike the case for the Leidenfrost drop on a flat substrate, for which only two regimes appear.

First, an outer region is identified, which is asymptotically the longest one of the three. Its longitudinal extent is comparable to the size of the drop. The vapour gap thickness in this region, scaling as $O\left(\tilde{\mathcal{E}}^{1 / 4}\right)$, only marginally varies relative to its value at the symmetry axis. This is quite different from the outer region in the vapour gap over a flat substrate, which appears in the form of a vapour pocket, wide in the centre and narrowing towards the edges (Snoeijer et al. 2009; Sobac et al. 2014), with a thickness scaling as $O\left(\tilde{\mathcal{E}}^{1 / 6}\right)$ (Sobac et al. 2014). It is such difference in morphology that is behind the expected suppression of the chimney instability for large Leidenfrost drops over a liquid pool, unlike their counterparts over a flat substrate (Snoeijer et al. 2009). An analytical solution for the thickness profile in the outer region was found in the case of large drops with the same liquid properties as the pool. In the case of smaller drops and/or differing properties, such a solution is expressed through numerically determined characteristics of the associated superhydrophobic drop.

Second, we identified an inner (neck) region at the exit from the vapour gap, the only one that bears a great resemblance to the corresponding region for Leidenfrost drops on a flat substrate (Snoeijer et al. 2009; Sobac et al. 2014). The neck thickness is here found to scale as $O\left(\tilde{\mathcal{E}}^{3 / 10}\right)$, which is close to the $O\left(\tilde{\mathcal{E}}^{1 / 3}\right)$ obtained for a flat substrate (Sobac et al. 2014). The longitudinal extent of the neck scales as $O\left(\tilde{\mathcal{E}}^{3 / 20}\right)$ (cf. $O\left(\tilde{\mathcal{E}}^{1 / 6}\right)$ for a flat substrate). A self-similar structure was found for the neck profile, which turns out the be governed by the same universal solution as for the Leidenfrost drop on a rigid substrate. The scaling laws and the self-similar shape showed excellent agreement with the full numerical solution.

Third, the peculiarities of the outer region morphology over a curved pool surface yet require the existence of an intermediate region to join the inner (neck) and outer ones. In contrast, no such intermediate region is present in the vapour gap over a flat substrate (Snoeijer et al. 2009; Sobac et al. 2014), where the outer and inner regions directly match one another. The vapour gap profile in this intermediate region turns out to be in the form of stationary capillary waves (undulations) springing from the neck and decaying towards the outer region. The largest bump, next to the neck, scales as $O\left(\tilde{\mathcal{E}}^{9 / 40}\right)$ in thickness, and $O\left(\tilde{\mathcal{E}}^{3 / 40}\right)$ in longitudinal extent. It is partly an excessive penetration of the undulations into the outer region that, for small but finite $\tilde{\mathcal{E}}$, limits the applicability of our asymptotic scheme as far as sufficiently small drops are concerned. 
On the one hand, the robustness of our analysis was tested in the realm of finitesized (but not too small) drops and differing liquids with a largely positive outcome, provided that the parameters are such that the pool surface remains essentially curved. On the other hand, the case of a large drop with the same liquid properties as the pool stands out as the ideal baseline for our asymptotic scheme and for which a large part of the analysis was carried out. Importantly, for these large Leidenfrost drops, pure scaling relations can be established in terms of the drop radius $R$ too, and not just in terms of $\tilde{\mathcal{E}}$ (a size-independent quantity). To recover their due form, it suffices to replace $\tilde{\mathcal{E}}$ in the earlier mentioned scaling relations with a modified (size-dependent) evaporation number defined in the present paper as $\mathcal{E}=6 \tilde{\mathcal{E}} R / \lambda_{c}$.

It is worth mentioning that the 'boules' described by Hickman (1964b), reproduced in figure 1, exhibit slightly different scaling laws. This is due to the different way the vapour is generated, which for the Hickman boules comes from the superheated pool. This is to be contrasted with 'usual' Leidenfrost case, for which the drop is evaporating. The main feature is that $t \sim \Delta T^{1 / 3}$ as opposed to $\Delta T^{1 / 4}$.

Finally, we found that the configuration of a flat substrate can here be recovered in the case of strongly differing liquid properties and hence a gradual change can in principle be realised between the two systems. However, as far as the details of a gradual transition between the asymptotic paradigms of flat and curved substrates are concerned, they remain a subject of future studies.

\section{Acknowledgements}

We thank one of the referees for pointing out a strong connection between the present work and the drainage problem. Part of this research has been funded by ESA-BELSPO via the Prodex Heat Transfer project, and by BELSPO via the IAP 7/38 MicroMAST network. B.S. and P.C. gratefully acknowledge financial support from the Fonds de la Recherche Scientifique - F.N.R.S. This work was partially supported by an ERC advanced grant.

\section{Appendix A. Numerical approach to the full problem}

Here we provide details of the numerical approach used to solve the full problem formulated in $\S 2.1$. In essence, the approach is similar to the one used by Sobac et al. (2014), Maquet et al. (2016), the modifications being largely of a geometric nature aimed at dealing with strongly curved substrates. We shall work in dimensionless terms by using $\lambda_{c}$ as the length scale and $\rho_{d} g \lambda_{c}=\gamma_{d} / \lambda_{c}$ as the pressure scale. The thereby obtained dimensionless variables are marked by hat, as e.g. in $(4.5 a-d)$.

The only exception to this hat rule is the drop radius $R$, for the dimensionless version of which we already have a notation $\mathcal{R}$, cf. (2.11). Nonetheless, expecting no confusion in the reader, the hats will be omitted in the remainder of this appendix for the sake of brevity, whereas they are still retained for the same quantities in the main body of the text.

\section{A.1. General equations}

Then equations (2.1), (2.2), (2.8) and (2.10) adopt the following dimensionless forms:

$$
\begin{gathered}
P_{v}=k-h-\kappa_{h} \quad \text { (everywhere), } \\
P_{v}=-\mathcal{P} e+\Gamma \kappa_{e} \quad \text { (everywhere), }
\end{gathered}
$$




$$
\begin{gathered}
P_{v}=0 \quad \text { (outside vapour layer) } \\
-\frac{1}{12} \partial_{s}\left[r t^{3} \partial_{s} P_{v}\right]=r \frac{\tilde{\mathcal{E}}}{t} \quad \text { (vapour layer). }
\end{gathered}
$$

When incorporating the latter (lubrication) equation in our numerical scheme, we shall aim at incurring a lubrication approximation error as low as $O\left(\delta^{2}\right)$ in terms of a thinfilm smallness parameter $\delta=1 / t \partial_{s} t$. This is normally automatically the case for a flat substrate. However, for a curved substrate (as the pool surface here), $O(\delta)$ errors may easily occur unless care is taken. We shall eventually use the following form of that equation:

$$
-\frac{1}{12} \partial_{s_{e}}\left[\frac{r_{h}+r_{e}}{2} t^{3}\left(1+\frac{1}{2} \kappa_{1 e} t\right) \partial_{s_{e}} P_{v}\right]=r_{h} \frac{\tilde{\mathcal{E}}}{t\left(1+\kappa_{1 e} t\right)} \quad \text { (vapour layer). }
$$

As used previously with $\kappa_{h}$ and $\kappa_{e}$, the subscripts ' $h$ ' and ' $e$ ' are used for the geometric quantities pertaining to the drop and pool surfaces, respectively, whereas $\kappa_{1}$ denotes the first curvature. As an independent variable, $s$, we have now chosen for definiteness the one along the pool surface, $s \equiv s_{e}$. The form of (A 5) can be understood using a differential identity $\mathrm{d} s_{e}=\mathrm{d} s_{h}\left(1+\kappa_{1 e} t\right)$ valid up to and including $O(\delta)$, where $\kappa_{1 e} t$ is an $O(\delta)$ correction with respect to unity. As the evaporation goes on from the drop surface while $s_{e}$ is the arc length along the pool surface, the evaporation term on the right-hand side is accordingly modified by a factor $\mathrm{d} s_{h} / \mathrm{d} s_{e}$. Besides, it is $r_{h}$ that is used there. In contrast, for the flux in the vapour film with a symmetric lubrication profile (no-slip conditions at both surfaces), it is most precise to base it on the middle surface (in between the drop and the pool). Hence there is a factor $\left(1+(1 / 2) \kappa_{1 e} t\right)$ (with a halved correction) modifying $\partial_{s_{e}} P_{v}$ and $\left(r_{h}+r_{e}\right) / 2$ for the radial coordinate on the left-hand side of (A 5). Note that the curvature quantities $\kappa$ are here defined as positive when the surfaces are concave towards the drop (see also below).

Equation (A 5) together with both (A 1) and (A 2) is applied inside the vapour layer, up until the patching point located at $s_{e}=s_{e, p a t c h}$. The choice of the patching point will be specified in more detail later on. Equation (A 3) is applied beyond the patching point to determine the equilibrium shapes of the upper part of the drop, together with (A 1), and of the remainder of the pool surface, together with (A 2).

The following geometric relations hold:

$$
\begin{array}{cccc}
\partial_{s_{h}} h=\sin \varphi_{h}, & \partial_{s_{h}} r_{h}=\cos \varphi_{h}, \quad \kappa_{h}=\kappa_{1 h}+\frac{\sin \varphi_{h}}{r_{h}}, \quad \kappa_{1 h}=\partial_{s_{h}} \varphi_{h}, \quad(\mathrm{~A} 6 a-d) \\
\partial_{s_{e}} e=\sin \varphi_{e}, & \partial_{s_{e}} r_{e}=\cos \varphi_{e}, \quad \kappa_{e}=\kappa_{1 e}+\frac{\sin \varphi_{e}}{r_{e}}, \quad \kappa_{1 e}=\partial_{s_{e}} \varphi_{e}, \quad(\mathrm{~A} 7 a-d)
\end{array}
$$

where $\varphi$ is the angle between the tangential along the surface pointing away from the axis underneath the drop and the horizontal pointing away from the axis. For definiteness, the vapour-layer thickness $t$ will be measured and the coordinate lines $s_{e}=$ const. will be defined exactly along the orthogonals to the pool surface. Then we can also write

$$
h-e=t \cos \varphi_{e}, \quad r_{e}-r_{h}=t \sin \varphi_{e}
$$

The arc length $s$ for each surface is counted from the axis underneath the drop, where we have $s_{e}=s_{h}=r_{e}=r_{h}=\varphi_{h}=\varphi_{e}=0$. We expect $\varphi_{h}=\pi$ at the axis at the top of the drop, whereas once again $\varphi_{e}=0$ at the unperturbed pool surface far away from the drop. 


\section{A.2. Upper part of the drop}

When solving for the upper, equilibrium part of the drop, one can get rid of the variable $s_{h}$ in the corresponding system of (A 1), (A 3) and (A 6) and render this part of the formulation in terms of an independent variable $\varphi_{h}$ (expected to vary monotonically in the interval of interest) and the dependent variables $h$ and $r_{h}$. We shall just shift the origin of $h$ to the top of the drop for later convenience. One obtains

$$
\partial_{\varphi_{h}} H=\frac{\sin \varphi_{h}}{\kappa_{h, t o p}-H-\sin \varphi_{h} / r_{h}}, \quad \partial_{\varphi_{h}} r_{h}=\frac{\cos \varphi_{h}}{\kappa_{h, t o p}-H-\sin \varphi_{h} / r_{h}},
$$

where

$$
h=h_{\text {top }}+H,
$$

with $h_{\text {top }}$ and $\kappa_{h, t o p}>0$ being the $h$ and curvature values, respectively, at the top of the drop. We also note that the constant $k$ appearing in (A 1) is hereby expressed as

$$
k=h_{\text {top }}+\kappa_{h, t o p} .
$$

The solution of the Cauchy problem (A9) with the boundary condition $H=0$ and $r_{h}=0$ at $\varphi_{h}=\pi$ (removable singularity in (A 9)) can be obtained by standard numerical methods, the integration proceeding towards $\varphi_{h}<\pi$.

The parameter $\kappa_{h, t o p}$ is actually the one determining the size (radius $\mathcal{R}$ ) of the drop. The formal equation on $\kappa_{h, t o p}$, once a system parameter $\mathcal{R}$ is specified, is

$$
\max _{0<\varphi_{h}<\pi} r_{h}\left(\varphi_{h}, \kappa_{h, t o p}\right)=\mathcal{R} .
$$

We note that in the cases when the patching point is located below the drop's equator (typically when the pool surface deformation is not too large), equation (A 12) can be solved immediately in the framework of the present equilibrium treatment of the upper part of the drop. Otherwise, equation (A 12) carries over to the vapour-layer problem to be considered in $\S$ A.4.

The parameter $h_{t o p}$, on the other hand, is the one determining the vertical shift of the drop, but not immediately affecting its shape. It is an unknown that carries over to the vapour-layer problem below.

For later use (see § A.4), to distinguish from the corresponding dependent variables in the vapour layer, we shall attribute a subscript 'upper' to the solution $H\left(\varphi_{h}, \kappa_{h, t o p}\right)$ and $r_{h}\left(\varphi_{h}, \kappa_{h, t o p}\right)$ obtained in the present subsection to yield

$$
H_{\text {upper }}\left(\varphi_{h}, \kappa_{h, t o p}\right) \text { and } r_{h, \text { upper }}\left(\varphi_{h}, \kappa_{h, t o p}\right) \text {, }
$$

presumed to be known functions in what follows and applied down to the patching point.

\section{A.3. Pool surface free from the drop}

Similarly to $\S$ A.2, equations (A 2), (A 3) and (A 7) describing the equilibrium shape of the pool surface beyond the patching point can be rendered in the form

$$
\partial_{\varphi_{e}} e=\frac{\sin \varphi_{e}}{\mathcal{P} \Gamma^{-1} e-\sin \varphi_{e} / r_{e}}, \quad \partial_{\varphi_{e}} r_{e}=\frac{\cos \varphi_{e}}{\mathcal{P} \Gamma^{-1} e-\sin \varphi_{e} / r_{e}} .
$$

Equations (A 14) are numerically integrated towards $\varphi_{e}>\varphi_{e 0}$ starting from numerics-adapted boundary conditions $e=C \mathrm{~K}_{0}\left(\mathcal{P}^{1 / 2} \Gamma^{-1 / 2} r_{e}\right)$ and $\varphi_{e}=-C \mathcal{P}^{1 / 2} \Gamma^{-1 / 2} \mathrm{~K}_{1}$ $\left(\mathcal{P}^{1 / 2} \Gamma^{-1 / 2} r_{e}\right)$ formulated at some numerically small $\varphi_{e}=\varphi_{e 0}$ (numerically large 
$\left.r_{e}=r_{e 0}\right)$. Here $\mathrm{K}_{0}$ and $\mathrm{K}_{1}$ are modified Bessel functions of the second kind. Such boundary conditions are inferred from (A 3) with (A2) by implying small pool surface deformations, when the curvature is given by $\kappa_{e}=\partial_{r_{e}}^{2} e+r_{e}^{-1} \partial_{r_{e}} e$, and the true boundary condition $e=0$ and $\varphi_{e}=0$ as $r_{e} \rightarrow \infty$. To the hereby obtained solution $e\left(\varphi_{e}, C\right)$ and $r_{e}\left(\varphi_{e}, C\right)$, we append for later convenience (as in $\S$ A.2) a distinctive subscript, here 'free', to yield

$$
e_{\text {free }}\left(\varphi_{e}, C\right) \text { and } r_{e, f r e e}\left(\varphi_{e}, C\right) \text {, }
$$

which are presumed to be known functions in what follows, applied down to the patching point. The constant $C$ is an unknown carrying over to the vapour-layer problem. We note that $e_{\text {free }}$ and $r_{\text {efree }}$ are also functions of a system parameter $\mathcal{P} \Gamma^{-1}$, but an explicit argument list shall here be limited to the variables and the unknown constants.

\section{A.4. Vapour layer}

When solving the problem in the vapour layer, we treat $s_{e}$ as an independent variable in the interval $0<s_{e}<s_{e, \text { patch }}$. Its value at the patching point, $s_{e, \text { patch }}$, is an unknown of the problem. Thirteen other variables entering the formulation (A 1), (A 2), (A 5)-(A 8) are taken as dependent ( $v i z . h, e, t, r_{h}, r_{e}, s_{h}, \varphi_{h}, \varphi_{e}, P_{v}, \kappa_{h}, \kappa_{e}, \kappa_{1 h}, \kappa_{1 e}$ ). Note the possibility of formally writing $\partial_{s_{h}}=\left(\partial_{s_{e}} s_{h}\right)^{-1} \partial_{s_{e}}$ in (A 6). For $k$ in (A 1), equation (A 11) is still implied.

Overall, we have an eighth-order ODE algebraic problem as written. The boundary conditions at $s_{e}=0$ are $s_{h}=r_{e}=\varphi_{e}=\varphi_{h}=\partial_{s_{e}} P_{v}=0\left(r_{h}=0\right.$ then formally following from the second algebraic equation (A 8)). At the patching point $s_{e}=s_{e, p a t c h}$, we have $P_{v}=0, h=H_{\text {upper }}\left(\varphi_{h}, \kappa_{h, \text { top }}\right)+h_{\text {top }}, r_{h}=r_{h, \text { upper }}\left(\varphi_{h}, \kappa_{h, \text { top }}\right), e=e_{\text {free }}\left(\varphi_{e}, C\right)$, $r_{e}=r_{e, f r e e}\left(\varphi_{e}, C\right)$, where the functions marked by the subscripts 'upper' and 'free' are regarded known from $\S$ A.2 and $\S$ A.3, respectively.

On the other hand, we still need a precise definition of the choice of the patching point, arbitrary within certain reasonable limits in our present scheme. We define it by setting the slope difference between the drop and pool surfaces at a sufficiently large prefixed value $\Delta \varphi$ (typically between $30^{\circ}$ and $90^{\circ}$ ), viz. $\varphi_{h}-\varphi_{e}=\Delta \varphi$ at $s_{e}=s_{e, p a t c h}$, which serves as yet another boundary condition. We verify a posteriori the results not being too sensitive to such a choice.

Thus, the number of the boundary conditions used (eleven) can be seen to exceed by three the differential order of the problem, which is justified given that there are also five unknown constants $\left(s_{e, p a t c h}, h_{\text {top }}, \kappa_{h, \text { top }}, k\right.$ and $\left.C\right)$ to be determined but so far only two equations, (A 11) and (A 12), formulated for them.

The vapour-layer problem is discretised by means of second-order finite differences at a uniform grid. The dependent variables are all defined only at the grid points themselves. The second-order ODE is discretised at the internal grid points, the first-order ODEs at the mid-points (the nonlinear terms being averaged between their values at the adjacent grid points), whereas the distributed algebraic equations hold at all grid points (due to a removable singularity in the third equations (A 6) and (A 7), they are replaced at the first grid point with $\kappa_{h}=2 \kappa_{1 h}$ and $\kappa_{e}=2 \kappa_{1 e}$ ). The boundary conditions are applied at the corresponding first or last grid points. The thereby obtained system of nonlinear algebraic equations for the values of the dependent variables at the grid points and the unknown constants, complemented yet 
by equations (A 11) and (A 12), is solved with the help of the FindRoot command in Mathematica, which finalises the solution for the vapour layer. On the other hand, as soon as the values of the constants $\left(s_{e, p a t c h}, h_{t o p}, \kappa_{h, t o p}\right.$ and $\left.C\right)$ are obtained, the shape of the upper part of the drop is eventually given by (A 13) applied for $\varphi_{h}$ in the interval $\left.\varphi_{h}\right|_{s_{e}=s_{e, p a t c h}}<\varphi_{h} \leqslant \pi$, where the definition (A 10) must be taken into account. The shape of the pool surface not covered by the drop is eventually given by (A 15) for $\varphi_{e}$ in the interval $0 \leqslant \varphi_{e}<\left.\varphi_{e}\right|_{s_{e}=s_{e, p a t c h}}$.

\section{Appendix B. Superhydrophobic drop computation}

In the present appendix, we proceed in the same non-dimensionalisation and with the same convention on hat omission as in the previous one (see the beginning of appendix A).

The solutions (A 13) and (A 15) for the upper part of the drop and for the dropfree part of the pool surface obtained in the context of a Leidenfrost drop actually hold verbatim in the context of a superhydrophobic drop. However, they must now be applied up until the contact (triple) line thereof, and not until a patching point, which is here non-existent. Equations (A 11) and (A 12) and the representation (A 10) are still applicable too.

For the drop-pool interface (the interfacial tension $\gamma_{d}+\gamma_{p}$ ) in the framework of our superhydrophobic drop, we have

$$
e \equiv h, \quad r_{e} \equiv r_{h}, \quad \varphi_{e} \equiv \varphi_{h}, \quad \kappa_{e} \equiv \kappa_{h} .
$$

The governing system of equations for this interface, a counterpart of (A 9) and (A 14) for the other two interfaces, can be derived by equating the expressions (A 1) and (A 2) on account of (B 1) and using geometric considerations similar to those used there. One finally arrives at

$$
\partial_{\varphi_{h}} \mathcal{H}=\frac{\sin \varphi_{h}}{\kappa_{h, \text { bottom }}-\frac{1-\mathcal{P}}{1+\Gamma} \mathcal{H}-\frac{\sin \varphi_{h}}{r_{h}}}, \quad \partial_{\varphi_{h}} r_{h}=\frac{\cos \varphi_{h}}{\kappa_{h, \text { bottom }}-\frac{1-\mathcal{P}}{1+\Gamma} \mathcal{H}-\frac{\sin \varphi_{h}}{r_{h}}},
$$

where

$$
h=h_{\text {bottom }}+\mathcal{H}
$$

and

$$
\kappa_{h, \text { bottom }}=\frac{\kappa_{h, \text { top }}+\left(h_{\text {top }}-h_{\text {bottom }}\right)+\mathcal{P} h_{\text {bottom }}}{1+\Gamma},
$$

the subscript 'bottom' referring to quantities at the very bottom of the drop (at the symmetry axis). The newly introduced quantities $h_{\text {bottom }}$ and $\kappa_{h \text {,bottom }}$ are unknowns to be determined from the overall problem.

The system (B 2) is numerically integrated starting from the boundary condition $\mathcal{H}=0$ and $r_{h}=0$ at $\varphi_{h}=0$ towards $\varphi_{h}>0$. To the hereby obtained solution $\mathcal{H}\left(\varphi_{h}, \kappa_{h, \text { bottom }}\right)$ and $r_{h}\left(\varphi_{h}, \kappa_{h, \text { bottom }}\right)$ we append for later distinction a subscript 'lower' to yield

$$
\mathcal{H}_{\text {lower }}\left(\varphi_{h}, \kappa_{h, \text { bottom }}\right) \text { and } r_{h, \text { lower }}\left(\varphi_{h}, \kappa_{h, \text { bottom }}\right),
$$

presumed to be known functions in what follows.

At the contact (triple) line, we must have continuity of all the three interfaces as well as of their corresponding slopes. On account of (A 10), (A 13), (A 15), (B 3) and (B 5), this leads to the following equations: 
where $\varphi_{C L}$ is the slope at the contact line (another unknown of the problem), common to all the three interfaces.

Finally, we have a system of seven algebraic equations, (A 11), (A 12), (B 4) and (B 6), for seven unknown constants, $k, h_{\text {top }}, \kappa_{h \text {,top }}, h_{\text {bottom }}, \kappa_{h \text {,bottom }}, C$ and $\varphi_{C L}$, which is solved numerically. Once the values of the constants are known and account is taken of the definitions (A 10) and (B 3), the eventual shape of the superhydrophobic drop and the adjacent free pool surface is given by (A 13) used for $\varphi_{C L} \leqslant \varphi_{h} \leqslant \pi$, (A 15) used for $0 \leqslant \varphi_{e} \leqslant \varphi_{C L}$, and (B 5) used for $0 \leqslant \varphi_{h} \leqslant \varphi_{C L}$. Once the shape is known, the vapour pressure (in an imaginary infinitesimal gap between the two liquids) is given either by (A 1) or, equivalently, by (A 2), hence a known distribution $P_{v}\left(\varphi_{h}\right)$. The arc length $s_{h}\left(\varphi_{h}\right)$ along the interface between the two liquids, required in the formulation of $\S 4$, can be determined by a posteriori integrating $\partial_{\varphi_{h}} s_{h}=\sqrt{\left(\partial_{\varphi_{h}} r_{h, \text { lower }}\right)^{2}+\left(\partial_{\varphi_{h}} \mathcal{H}_{\text {lower }}\right)^{2}}$ and implying $s_{h}=0$ at the symmetry axis. In this way, we can express $P_{v}$ and $r_{h \text {,lower }}$ as functions of $s_{h}$, which we symbolically rewrite below in the notations to be used in $\S 4$ :

$$
\hat{P}_{v}(\hat{s}), \quad \hat{r}(\hat{s}),
$$

i.e. with a hat restored (cf. the beginning of appendix A) and subscripts ' $h$ ' and 'lower' dropped. The value of this arc length at the contact line, $s_{h}\left(\varphi_{C L}\right)$, will likewise be denoted as $\hat{s}_{C L}$ in $\S 4$.

\section{REFERENCES}

Baumeister, K. J. \& Simon, F. F. 1973 Leidenfrost temperature - its correlation for liquid metals, cryogens, hydrocarbons, and water. Trans. ASME J. Heat Transfer 95 (2), 166-173.

BeJan, A. 1993 Heat Transfer, 1st edn. Wiley.

Benilov, E. S., Chapman, S. J., Mcleod, J. B., Ockendon, J. R. \& Zubkov, V. S. 2010 On liquid films on an inclined plate. J. Fluid Mech. 663, 53-69.

Bernardin, J. D. \& Mudawar, I. 1999 The Leidenfrost point: experimental study and assessment of existing models. Trans. ASME J. Heat Transfer 121, 894-903.

Biance, A.-L., Clanet, C. \& Quéré, D. 2003 Leidenfrost drops. Phys. Fluids 15 (6), 1632-1637.

Bouwhuis, W., Winkels, K. G., Peters, I. R., Brunet, P., van Der Meer, D. \& Snoeijer, J. H. 2013 Oscillating and star-shaped drops levitated by an airflow. Phys. Rev. E 88, 023017.

BRUnet, P. \& SNOEIJER, J. H. 2011 Star-drops formed by periodic excitation and on an air cushion - a short review. Eur. Phys. J. Spec. Top. 192 (1), 207-226.

Burton, J. C., Sharpe, A. L., van Der Veen, R. C. A., Franco, A. \& Nagel, S. R. 2012 Geometry of the vapor layer under a Leidenfrost drop. Phys. Rev. Lett. 109 (7), 074301.

Celestini, F., Frish, T. \& Pomeau, Y. 2012 Take off of small Leidenfrost droplets. Phys. Rev. Lett. 109, 034501.

Chandra, S. \& Avedisian, C. T. 1991 On the collision of a droplet with a solid surface. Proc. R. Soc. Lond. A 432 (1884), 13-41.

De Gennes, P.-G., Brochard-Wyart, F. \& Quéré, D. 2013 Capillarity and Wetting Phenomena: Drops, Bubbles, Pearls, Waves. Springer Science \& Business Media.

Duchemin, L., Lister, J. R. \& LAnge, U. 2005 Static shapes of levitated viscous drops. J. Fluid Mech. 533, 161-170.

Hickman, K. C. D. $1964 a$ Floating drops and boules. Nature 201 (4923), 985.

Hickman, K. C. D. $1964 b$ Floating drops and liquid boules. Ind. Engng Chem. 56 (6), 18-31.

Hinch, E. J. \& Lemaitre, J. 1994 The effect of viscosity on the height of disks floating above an air table. J. Fluid Mech. 273, 313-322.

Holter, N. J. \& Glasscock, W. R. 1952 Vibrations of evaporating liquid drops. J. Acoust. Soc. Am. 24 (6), 682-686. 
Janssens, S. D., Koizumi, S. \& Fried, E. 2017 Behavior of self-propelled acetone droplets in a Leidenfrost state on liquid substrates. Phys. Fluids 29 (3), 032103.

Jones, A. F. \& WiLson, S. D. R. 1978 The film drainage problem in droplet coalescence. J. Fluid Mech. 87 (2), 263-288.

Lagubeau, G., Le Merrer, M., Clanet, C. \& Quéré, D. 2011 Leidenfrost on a ratchet. Nat. Phys. 7 (5), 395-398.

Landau, L. D. \& Lifshitz, E. M. 1959 Fluid Mechanics, Course of Theoretical Physics, vol. 6. Pergamon.

Leidenfrost, J. G. 1756 De Aquae Communis Nonnullis Qualitatibus Tractatus. Ovenius.

van Limbeek, M. A. J., Shirota, M., Sleutel, P., Sun, C., Prosperetti, A. \& Lohse, D. 2016 Vapour cooling of poorly conducting hot substrates increases the dynamic Leidenfrost temperature. Intl J. Heat Mass Transfer 97, 101-109.

Linke, H., Alemán, B. J., Melling, L. D., Taormina, M. J., Francis, M. J., Dow-Hygelund, C. C., Narayanan, V., Taylor, R. P. \& Stout, A. 2006 Self-propelled Leidenfrost droplets. Phys. Rev. Lett. 96 (15), 154502.

Lister, J. R., Thompson, A. B., Perriot, A. \& Duchemin, L. 2008 Shape and stability of axisymmetric levitated viscous drops. J. Fluid Mech. 617, 167-185.

Ma, X., Liétor-Santos, J.-J. \& Burton, J. C. 2017 Star-shaped oscillations of Leidenfrost drops. Phys. Rev. Fluids 2, 031602.

Maquet, L., Sobac, B., Darbois-Texier, B., Duchesne, A., Brandenbourger, M., Rednikov, A., Colinet, P. \& Dorbolo, S. 2016 Leidenfrost drops on a heated liquid pool. Phys. Rev. Fluids 1, 053902.

QuÉRÉ, D. 2013 Leidenfrost dynamics. Annu. Rev. Fluid Mech. 45, 197-215.

RYUji, T. \& Ken, A. 1985 Vibration of a flattened drop. II. Normal mode analysis. J. Phys. Soc. Japan 54 (7), 2462-2469.

Shirota, M., van Limbeek, M. A. J., Sun, C., Prosperetti, A. \& Lohse, D. 2016 Dynamic Leidenfrost effect: relevant time and length scales. Phys. Rev. Lett. 116, 064501.

SnezhKo, A., Ben JaCob, E. \& ARAnson, I. S. 2008 Pulsating-gliding transition in the dynamics of levitating liquid nitrogen droplets. New J. Phys. 10 (4), 043034.

Snoeijer, J. H., Brunet, P. \& EgGers, J. 2009 Maximum size of drops levitated by an air cushion. Phys. Rev. E 79, 036307.

Snoeijer, J. H., Ziegler, J., Andreotti, B., Fermigier, M. \& Eggers, J. 2008 Thick films of viscous fluid coating a plate withdrawn from a liquid reservoir. Phys. Rev. Lett. 100 (24), 244502.

Sobac, B., Rednikov, A., Dorbolo, S. \& Colinet, P. 2014 Leidenfrost effect: accurate drop shape modeling and refined scaling laws. Phys. Rev. E 90 (5), 053011.

Sobac, B., Rednikov, A., Dorbolo, S. \& Colinet, P. 2017 Self-propelled Leidenfrost drops on a thermal gradient: a theoretical study. Phys. Fluids 29, 082101.

Strier, D. E., Duarte, A. A., Ferrari, H.\& Mindlin, G. B. 2000 Nitrogen stars: morphogenesis of a liquid drop. Physica A 283 (1-2), 261-266.

Tran, T., Staht, H. J. J., Prosperetti, A., Sun, C. \& Lohse, D. 2012 Drop impact on superheated surfaces. Phys. Rev. Lett. 108 (3), 036101.

Wachters, L. H. J, Bonne, H. \& VAn Nouhuis, H. J. 1966 The heat transfer from a hot horizontal plate to sessile water drops in the spherodial state. Chem. Engng Sci. 21, 923-936.

Weisstein, E. 2017 Hypergeometric function. In Wolfram MathWorld, Wolfram Web Resources.

Wilson, S. D. R. \& Jones, A. F. 1983 The entry of a falling film into a pool and the airentrainment problem. J. Fluid Mech. 128, 219-230.

WÜRger, A. 2011 Leidenfrost gas ratchets driven by thermal creep. Phys. Rev. Lett. 107 (16), 164502.

YiAntsios, S. G. \& DAVIS, R. H. 1990 On the buoyancy-driven motion of a drop towards a rigid surface or a deformable interface. J. Fluid Mech. 217, 547-573. 